\title{
Adaptation bounds for confidence bands under self-similarity
}

\author{
TIMOTHY B. ARMSTRONG \\ Department of Economics, Yale University, 30 Hillhouse Ave., New Haven, CT 06511, USA. \\ E-mail: timothy.armstrong@yale.edu
}

We derive bounds on the scope for a confidence band to adapt to the unknown regularity of a nonparametric function that is observed with noise, such as a regression function or density, under the self-similarity condition proposed by Giné and Nickl (Ann. Statist. 38 (2010) 1122-1170). We find that adaptation can only be achieved up to a term that depends on the choice of the constant used to define self-similarity, and that this term becomes arbitrarily large for conservative choices of the self-similarity constant. We construct a confidence band that achieves this bound, up to a constant term that does not depend on the self-similarity constant. Our results suggest that care must be taken in choosing and interpreting the constant that defines self-similarity, since the dependence of adaptive confidence bands on this constant cannot be made to disappear asymptotically.

Keywords: Honest confidence interval; adaptation; self-similarity

\section{Introduction}

Consider the problem of constructing a confidence band for a function that is observed with noise, such as a regression function or density. It will be convenient to state our results in the white noise model

$$
Y(t)=\int_{0}^{t} f(s) d s+\sigma_{n} W(t), \quad \sigma_{n}=\sigma / \sqrt{n},
$$

which maps to the regression or density setting with $n$ playing the role of sample size [3,24]. Here $f: \mathbb{R} \rightarrow \mathbb{R}$ is an unknown function, $W(t)$ is a standard Brownian motion and $Y(t)$ is observed with $\sigma_{n}$ treated as known. To obtain good estimates and confidence bands, one must impose some regularity on the function $f$. This is typically done by assuming that $f$ is in a derivative smoothness class, such as the Hölder class $\mathcal{F}_{\text {Höl }}(\gamma, B)$, which formalizes the notion that the $\gamma$ th derivative is bounded by $B$ :

$$
\mathcal{F}_{\text {Höl }}(\gamma, B)=\left\{f: \text { for all } t, t^{\prime} \in \mathbb{R},\left|f^{(\lfloor\gamma\rfloor)}(t)-f^{(\lfloor\gamma\rfloor)}\left(t^{\prime}\right)\right| \leq B\left|t-t^{\prime}\right|^{\gamma-\lfloor\gamma\rfloor}\right\},
$$

where $\lfloor\gamma\rfloor$ denotes the greatest integer strictly less than $\gamma$. We are interested in constructing a confidence band for $f$ on an interval, which we take to be [0,1]. A confidence band is a collection of random intervals $\mathcal{C}_{n}(x)=\mathcal{C}_{n}(x ; Y)$ for $x \in[0,1]$ that depend on the data $Y$ observed at noise level $\sigma_{n}=\sigma / \sqrt{n}$. Following the standard definition, we say that $\mathcal{C}_{n}(\cdot)$ is a confidence band with coverage $1-\alpha$ over the class $\mathcal{F}$ if

$$
\inf _{f \in \mathcal{F}} P_{f}\left(\text { for all } x \in[0,1], f(x) \in \mathcal{C}_{n}(x)\right) \geq 1-\alpha,
$$

where $P_{f}$ denotes probability when $Y(t)$ is drawn according to $f$. Although we focus on the interval $[0,1]$, to avoid boundary issues, we will assume that $Y(t)$ is observed on an interval $[-\eta, 1+\eta]$ for some $\eta>0$. 
Using knowledge of the class $\mathcal{F}_{\text {Höl }}(\gamma, B)$, one can construct estimators and confidence bands that are near-optimal in a minimax sense. In practice, however, it can be difficult to specify $\gamma$ and $B$ a priori. This has led to the paradigm of adaptation: one seeks estimators and confidence bands that are nearly optimal for all $\gamma$ and $B$ in some range without a priori knowledge of $\gamma$ or $B$. Such procedures are called "adaptive." Unfortunately, while it is possible to construct estimators that adapt to the unknown value of $\gamma$ and $B$, (see [31] and references therein), it follows from [20] that adaptive confidence band construction over derivative smoothness classes is impossible.

To recover the possibility of adaptive confidence band construction, [12] propose an additional condition known as "self-similarity" (see also [26]), which uses a constant $\varepsilon>0$ to rule out functions such that the level of regularity is statistically difficult to detect. Imposing these additional conditions leads to a class $\mathcal{F}_{\text {self-sim }}(\gamma, B, \varepsilon) \subsetneq \mathcal{F}_{\text {Höl }}(\gamma, B)$. [12] derive confidence bands that are rate-adaptive to the unknown parameter $\gamma$ over these smaller classes, and they show that the set $\mathcal{F}_{\text {Höl }}(\gamma, B) \backslash \bigcup_{\varepsilon>0} \mathcal{F}_{\text {self-sim }}(\gamma, B, \varepsilon)$ of functions ruled out by this assumption (as $\varepsilon \rightarrow 0$ ) is small in a certain topological sense. A subsequent literature has further examined the use of self-similarity and related assumptions in forming adaptive confidence bands (see references below).

These results provide a promising approach to constructing a confidence band such that the width reflects the unknown regularity $\gamma$ of the function $f$. However, these confidence bands require a priori knowledge of other regularity parameters, including $\varepsilon$, either explicitly or through unspecified constants and sequences that must be chosen in a way that depends on $\varepsilon$ in order to guarantee coverage for a given sample size or noise level. Furthermore, these choices have a first order asymptotic effect on the width of the confidence band, and making an asymptotically conservative choice by taking $\varepsilon=\varepsilon_{n} \rightarrow 0$ leads to a slightly slower rate of convergence. This has led to concern about whether self-similarity assumptions can lead to a "practical" approach to confidence band construction (see, for example, the discussion on pp. 2388-2389 of [14]): while self-similarity removes the need to specify the order $\gamma$ of the derivative, currently available methods still require specifying other regularity parameters. Can one construct a confidence band that is fully adaptive without specifying any of the regularity parameters $\gamma, B$ or $\varepsilon$ ?

An implication of the results in this paper is that it is impossible to achieve such a goal. In particular, we show that a confidence band that is adaptive over classes $\mathcal{F}_{\text {self-sim }}(\gamma, B, \varepsilon)$ over a range of $\gamma$ or $B$ must necessarily pay an adaptation penalty proportional to $\varepsilon^{-1 /(2 \gamma+1)}$. As a consequence, adaptive confidence bands in self-similarity classes require explicit specification of the self-similarity constant $\varepsilon$, and taking $\varepsilon=\varepsilon_{n} \rightarrow 0$ requires paying a penalty in the rate. On a more positive note, once $\varepsilon$ is given, we construct a confidence band that is "practical" in the sense that it is valid for a fixed sample size or noise level in Gaussian settings, and it does not depend on additional unspecified constants or sequences once $\varepsilon$ is given.

To describe these results formally, let $\mathcal{I}_{n, \alpha, \mathcal{F}}$ denote the set of confidence bands that satisfy the coverage requirement (1). Subject to this coverage requirement, we compare worst-case length of $\mathcal{C}_{n}$ over a possibly smaller class $\mathcal{G}$. Letting length $(\mathcal{A})=\sup \mathcal{A}-\inf \mathcal{A}$ denote the length of a set $\mathcal{A}$, let

$$
R_{\beta}\left(\mathcal{C}_{n} ; \mathcal{G}\right)=\sup _{f \in \mathcal{G}} q_{\beta, f}\left(\sup _{x \in[0,1]} \operatorname{length}\left(\mathcal{C}_{n}(x)\right)\right),
$$

where $q_{\beta, f}$ denotes the $\beta$ quantile when $Y \sim f$. Following [7], define

$$
R_{n, \alpha, \beta}^{*}(\mathcal{G}, \mathcal{F})=\inf _{\mathcal{C}_{n}(\cdot) \in \mathcal{I}_{n, \alpha, \mathcal{F}}} R_{\beta}\left(\mathcal{C}_{n} ; \mathcal{G}\right)
$$

to be the optimal worst-case length over $\mathcal{G}$ of a band with coverage over $\mathcal{F}$, where $\mathcal{G} \subseteq \mathcal{F}$. A minimax confidence band over the set $\mathcal{F}$ is one that achieves the bound $R_{n, \alpha, \beta}^{*}(\mathcal{F}, \mathcal{F})$. Given a family $\mathcal{F}(\tau)$ 
of function classes indexed by a regularity parameter $\tau \in \mathcal{T}$, the goal of adaptive confidence band construction is to find a single confidence band $\mathcal{C}_{n}(\cdot)$ that is close to achieving this bound for each $\mathcal{F}(\tau)$, while also maintaining coverage $1-\alpha$ for each $\mathcal{F}(\tau)$ (so that $\mathcal{C}_{n}(\cdot) \in \mathcal{I}_{n, \alpha, \bigcup_{\tau \in \mathcal{T}}} \mathcal{F ( \tau )}$ ). Suppose that a confidence band $\mathcal{C}_{n}(\cdot) \in \mathcal{I}_{n, \alpha, \bigcup_{\tau \in \mathcal{T}}} \mathcal{F}(\tau)$ achieves this goal up to a factor $A_{n}(\tau)$ :

$$
R_{\beta}\left(\mathcal{C}_{n} ; \mathcal{F}(\tau)\right) \leq A_{n}(\tau) R_{n, \alpha, \beta}^{*}(\mathcal{F}(\tau), \mathcal{F}(\tau)) \quad \text { all } \tau \in \mathcal{T}
$$

(in the present setting, $A_{n}(\tau)$ will not depend on $\alpha$ or $\beta$ once $n$ is large enough). We will call such a band adaptive to $\tau$ up to the adaptation penalty $A_{n}(\tau)$. If the adaptation penalty is bounded as a function of $n$, we will say that the confidence band is (rate) adaptive (this corresponds to what [7] call "strongly adaptive"). Note that $R_{n, \alpha, \beta}^{*}\left(\mathcal{F}(\tau), \bigcup_{\tau \in \mathcal{T}} \mathcal{F}(\tau)\right) / R_{n, \alpha, \beta}^{*}(\mathcal{F}(\tau), \mathcal{F}(\tau))$ provides a lower bound for the adaptation penalty of any confidence band $\mathcal{C}_{n}(\cdot)$.

For Hölder classes, $R_{n, \alpha, \beta}^{*}\left(\mathcal{F}_{\text {Höl }}(\gamma, B), \mathcal{F}_{\text {Höl }}(\gamma, B)\right)$ decreases at the rate $(n / \log n)^{-\gamma /(2 \gamma+1)}$. A confidence band that is rate adaptive to $\gamma$ would achieve this rate simultaneously for all $\gamma$ in some set $[\underline{\gamma}, \bar{\gamma}]$ while maintaining coverage over $\bigcup_{\gamma \in[\underline{\gamma}, \bar{\gamma}]} \mathcal{F}_{\text {Höl }}(\gamma, B)$. However, as noted above, the results of [20] imply that this is impossible. Indeed, $R_{n, \alpha, \beta}^{*}\left(\mathcal{F}_{\text {Höl }}(\gamma, B), \bigcup_{\gamma^{\prime} \in[\underline{\gamma}, \bar{\gamma}]} \mathcal{F}_{\text {Höl }}\left(\gamma^{\prime}, B\right)\right)$ decreases at the rate $(n / \log n)^{-\underline{\gamma} /(2 \underline{\gamma}+1)}$ for each $\gamma \in[\underline{\gamma}, \bar{\gamma}]$, so the adaptation penalty for Hölder classes is of order $(n / \log n)^{\gamma /(2 \gamma+1)-\underline{\gamma} /(2 \underline{\gamma}+1)}$, which is quite severe.

To salvage the possibility of adaptation, [12] propose augmenting the Hölder condition with an auxiliary condition. Let $K: \mathbb{R}^{2} \rightarrow \mathbb{R}$ be a function, called a kernel, such that $x \mapsto K(t, x)$ is of bounded variation for each $t$. Let $K_{j}(t, x)=2^{j} K\left(2^{j} t, 2^{j} x\right)$ for any integer $j$, and let $\hat{f}(t, j)=\int K_{j}(t, x) d Y(x)$. This allows for convolution kernels $K(t, x)=\tilde{K}(t-x)$ (in which case $2^{-j}$ is the bandwidth) and wavelet projection kernels $K(t, x)=\sum_{k} \phi(t-k) \phi(x-k)$ (in which case $\phi$ is the father wavelet and $j$ is the resolution level). Let $K_{j} f(t)=\int K_{j}(t, x) f(x) d x$. Note that $E_{f} \hat{f}(t, j)=K_{j} f(t)$, where $E_{f}$ denotes expectation when $Y(x)$ is drawn according to $f$, so that the bias is given by $K_{j} f(t)-f(t)$. Under appropriate conditions on $K$, an upper bound on this bias for functions in $\mathcal{F}_{\text {Höl }}(\gamma, B)$ follows from standard calculations (see [13], Ch. 4):

$$
\sup _{t \in[0,1]}\left|K_{j} f(t)-f(t)\right| \leq \tilde{C} B 2^{-j \gamma}
$$

for some constant $\tilde{C}$. [12] impose such a bound on bias directly, along with an analogous lower bound. For $j, b_{1}, b_{2}>0$, let $\mathcal{F}_{\mathrm{GN}}\left(\gamma, b_{1}, b_{2}\right)=\mathcal{F}_{\mathrm{GN}}\left(\gamma, b_{1}, b_{2} ; K, \underline{j}\right)$ denote the set of functions $f$ satisfying Condition 3 of [12]: for all integers $j \geq \underline{j}$,

$$
b_{1} 2^{-j \gamma} \leq \sup _{t \in[0,1]}\left|K_{j} f(t)-f(t)\right| \leq b_{2} 2^{-j \gamma}
$$

Since we will also be imposing Hölder conditions, which, as noted above, satisfy the upper bound with $b_{2}=\tilde{C} B$, it is natural to make the lower bound proportional to $B$ as well, by taking $b_{1}=\varepsilon B$ for some $\varepsilon>0$. To this end, let $\mathcal{F}_{\text {self-sim }}(\gamma, B, \varepsilon)=\mathcal{F}_{\text {self-sim }}(\gamma, B, \varepsilon ; K, \underline{j})$ be the set of functions in $\mathcal{F}_{\text {Höl }}(\gamma, B)$ such that the lower bound in (3) holds with $b_{1}=\varepsilon B$ for all integers $j \geq \underline{j}$. By the discussion above, this is equivalent to defining $\mathcal{F}_{\text {self-sim }}(\gamma, B, \varepsilon ; K, j)=\mathcal{F}_{\text {Höl }}(\gamma, B) \cap \mathcal{F}_{\mathrm{GN}}(\gamma, \varepsilon B, C B ; K, j)$ for any $C \geq \tilde{C}$. We will refer to $\varepsilon$ as a "self-similarity constant," and we will call the class $\mathcal{F}_{\text {self-sim }}$ a "self-similarity class." Note that, by defining $\varepsilon$ to be (up to a constant) the ratio of the upper and lower bounds on the bias, we are separating the role of self-similarity and the smoothness constant. In particular, the self-similarity constant is scale invariant. See Section 2.3 for alternative formulations of the notion of a "self-similarity constant." 
Our main results are efficiency bounds that have implications for the adaptation penalty $A_{n}(\gamma, B)$ for confidence bands that adapt to the regularity parameters $(\gamma, B)$ over a rich enough set $\mathcal{T}$ in the selfsimilarity class $\mathcal{F}_{\text {self-sim }}(\varepsilon, \gamma, B)$. In particular, our results imply the existence of a constant $C_{*}>0$ such that, for large enough $n$, the adaptation penalty for any confidence band must satisfy the lower bound $C_{*} \varepsilon^{-1 /(2 \gamma+1)}<A_{n}(\gamma, B)$. Furthermore, we construct a confidence band with adaptation penalty $A_{n}(\gamma, B)<C^{*} \varepsilon^{-1 /(2 \gamma+1)}$, where $C^{*}<\infty$ (the constants $C_{*}$ and $C^{*}$ do not depend on $\varepsilon$ but may depend on the set $\mathcal{T}$ over which adaptation is required). For the lower bounds, we consider separately the cases of adaptation to $B$ with $\gamma$ known (i.e., $\mathcal{T}=\gamma \times[\underline{B}, \bar{B}]$ ) and adaptation to $\gamma$ with $B$ known (i.e., $\mathcal{T}=[\gamma, \bar{\gamma}] \times B$ ). In both cases, the lower bound gives the same $\varepsilon^{-1 /(2 \gamma+1)}$ term. We also consider the possibility of "adapting to the self-similarity constant" and find that that this is not possible: if we allow $\varepsilon$ to be in some set $[\underline{\varepsilon}, \bar{\varepsilon}]$, then we obtain a lower bound proportional to $\underline{\varepsilon}^{-1 /(2 \gamma+1)}$.

Our results relate to the literature deriving confidence bands under self-similarity conditions. [12] propose a confidence band that has coverage over $f \in \mathcal{F}_{\text {self-sim }}\left(\gamma, B, \varepsilon_{n}\right)$ for a range of $(\gamma, B)$, where $\varepsilon_{n} \rightarrow 0$ with the sample size, and they show that it is adaptive up to a penalty $A_{n}(\gamma, B)$ where $A_{n}(\gamma, B) \rightarrow \infty$ slowly with the sample size $n$. Our lower bounds show that a penalty of this form is unavoidable if one takes $\varepsilon_{n} \rightarrow 0$. [5] and [10] propose confidence bands with coverage over selfsimilarity classes with $\varepsilon$ fixed, and they show that these confidence bands are fully rate adaptive (i.e., the adaptation penalty $A_{n}(\gamma, B)$ is bounded as $n$ increases). Checking whether the adaptation penalty for these confidence bands takes the optimal form $C^{*} \varepsilon^{-1 /(2 \gamma+1)}$ for small $\varepsilon$ appears to be difficult, and we derive upper bounds using a different confidence band (although the confidence band, we propose builds on ideas in these papers; see Section 2.4).

To our knowledge, this paper is the first to derive lower bounds on adaptation constants for confidence bands under self-similarity conditions. A related question, addressed by [14] and [5], is whether the self-similarity conditions themselves can be weakened. These papers derive lower bounds showing that certain ways of relaxing self-similarity necessarily lead to a penalty in the rate, and our finding that taking $\varepsilon=\varepsilon_{n} \rightarrow 0$ requires paying such a penalty complements these results. In addition, a large literature has considered adaptive confidence sets in related settings under conditions that are similar to the self-similarity condition used by [12]. In the Gaussian sequence setting, [30] propose a condition called a "polished tail" condition. They use this condition to show frequentist coverage of adaptive Bayesian credible sets (see also $[29,32]$ ). Other applications of self-similarity type conditions include high dimensional sparse regression [23], density estimation on the sphere [18], locally adaptive confidence bands [25], binary regression [21] and $L_{p}$ confidence sets [6,9,22] (in contrast to our setting where $p=\infty$, some range of adaptation is possible even without self-similarity when $p<\infty$; see $[8,16,28]$ ). Self-similarity is also related to "signal strength" conditions used in other settings, such as "beta-min" conditions used to study variable selection in high dimensional regression (see [4], Section 7.4).

Our lower bounds apply immediately to confidence bands with coverage under any set $\mathcal{F}$ that weakens the self-similarity conditions in [12]. This includes, for certain ranges of regularity constants, the conditions used in [5] and, for adaptation to $B$ with $\gamma$ fixed, [14]. [30] show that their conditions are weaker than a natural definition of self-similarity in the Gaussian sequence setting. A full characterization of upper and lower bounds in these and other related settings is left for future research.

\section{Adaptation bounds for self-similar functions}

This section states our main results. We first give lower bounds for adaptation, separating the role of adaptation to the constant $B$ and the exponent $\gamma$. We then construct a confidence band that achieves these bounds, up to a constant that does not depend on the self-similarity constant $\varepsilon$, simultaneously for all $\gamma$ and $B$ on bounded intervals. Finally, we provide lower bounds for an alternative formulation of the problem, and a discussion of our results. 
Before stating the formal results, we give a heuristic explanation of the bounds. Self-similarity allows for adaptation by bounding the bias at a scale $j_{1}$ using an estimate of the bias at a different scale $j_{2}$ : the bias $\sup _{t \in[0,1]}\left|K_{j_{1}} f(t)-f(t)\right|$ of $\hat{f}\left(t, j_{1}\right)$ is bounded by $\varepsilon^{-1} \tilde{C} 2^{-\gamma\left(j_{1}-j_{2}\right)} \sup _{t \in[0,1]}\left|K_{j_{2}} f(t)-f(t)\right|$. If we can get an estimate of this upper bound that converges more quickly than the estimation error in $\hat{f}\left(t, j_{1}\right)$ (which turns out to be possible by taking $j_{2}$ to increase slightly more slowly than $j_{1}$ ), then we can treat this upper bound as known. Since $\sup _{t \in[0,1]}\left|K_{j_{2}} f(t)-f(t)\right|$ is bounded by $\tilde{C} B 2^{-\gamma j_{2}}$, this is as good as using the bound $\varepsilon^{-1} \tilde{C}^{2} B 2^{-\gamma j_{1}}$ on the bias of $\hat{f}\left(t, j_{1}\right)$. Choosing $j_{1}$ to balance this term with the estimation error in $\sup _{t \in[0,1]}\left|\hat{f}\left(t, j_{1}\right)-K_{j_{1}}(f)\right|$ then gives the rate with the $\varepsilon^{-1 /(2 \gamma+1)}$ factor. Note that this gives the same rate and constant as using prior knowledge of the Hölder class, but replacing $B$ with $\varepsilon^{-1} B$, up to a constant that does not depend on $\varepsilon, \gamma$ or $B$.

The constructive upper bound in Section 2.2 below uses a confidence band that formalizes these ideas. The lower bounds in Section 2.1 show formally that no further information can be used to improve this confidence band, up to factors that do not depend on $\varepsilon, \gamma$ or $B$.

\subsection{Lower bounds}

We now give bounds for adaptation over the classes $\mathcal{F}_{\text {self-sim }}(\gamma, B, \varepsilon)$. Proofs of the lower bounds in this section are given in Section 3. We impose the following conditions on the kernel $K$ :

$$
\begin{aligned}
& \text { there exists } C_{K}<\infty \text { such that } K(y, x)=0 \text { for }|x-y|>C_{K} \text { and, for all } k \in \mathbb{Z} \\
& \text { and } x, y \in \mathbb{R}, K(y, x)=K(y-k, x-k) \text {. }
\end{aligned}
$$

These conditions hold for convolution kernels with finite support, and for wavelet projection kernels for which the father wavelet has bounded support.

We first consider adaptation to the constant $B$.

Theorem 2.1. Let $\gamma>0$ and let $0<2 \alpha<\beta<1$. Let $K$ be a kernel satisfying (4). There exists $\underline{j}_{K, \gamma}$, $C_{K, \gamma, *}>0$ and $\eta_{K, \gamma}>0$ such that, for any $0<\underline{B} \leq B \leq \bar{B}, \varepsilon \leq \varepsilon^{\prime}<\eta_{K, \gamma}$ and $\underline{\ell}^{2} \underline{j}_{K, \gamma}$,

$$
\begin{aligned}
& R_{n, \alpha, \beta}^{*}\left(\mathcal{F}_{\text {self-sim }}\left(\gamma, B, \varepsilon^{\prime} ; K, \underline{\ell}\right), \bigcup_{B^{\prime} \in[\underline{B}, \bar{B}]} \mathcal{F}_{\text {self-sim }}\left(\gamma, B^{\prime}, \varepsilon ; K, \underline{\ell}\right)\right) \\
& \geq(1+o(1)) C_{K, \gamma, *} \min \left\{\varepsilon^{-1} B, \bar{B}\right\}^{1 /(2 \gamma+1)}\left(\sigma_{n}^{2} \log \left(1 / \sigma_{n}\right)\right)^{\gamma /(2 \gamma+1)} .
\end{aligned}
$$

We now consider adaptation to $\gamma$ with $B$ known. To avoid notational clutter, we normalize $B$ to one.

Theorem 2.2. Let $0<\gamma<\gamma \leq \bar{\gamma}$ and let $0<2 \alpha<\beta<1$. Let $K$ be a kernel that satisfies (4). There exist $C_{K, \bar{\gamma}, *}, \underline{j}_{K, \bar{\gamma}}$ and $\bar{\eta}_{K, \bar{\gamma}}$ depending only on $K$ and $\bar{\gamma}$ such that, for all $\underline{\ell}^{\geq} \underline{j}_{K, \bar{\gamma}}$ and $0<\varepsilon \leq \varepsilon^{\prime}<$ $\eta_{K, \bar{\gamma}}$

$$
\begin{gathered}
R_{n, \alpha, \beta}^{*}\left(\mathcal{F}_{\text {self-sim }}\left(\gamma, 1, \varepsilon^{\prime} ; K, \underline{\ell}\right), \bigcup_{\gamma^{\prime} \in[\underline{\gamma}, \bar{\gamma}]} \mathcal{F}_{\text {self-sim }}\left(\gamma^{\prime}, 1, \varepsilon ; K, \underline{\ell}\right)\right) \\
\geq(1+o(1)) C_{K, \bar{\gamma}, *} \varepsilon^{-1 /(2 \gamma+1)}\left(\sigma_{n}^{2} \log \left(1 / \sigma_{n}\right)\right)^{\gamma /(2 \gamma+1)} .
\end{gathered}
$$

It follows from Theorems 2.1 and 2.2 that adaptive confidence bands must pay an adaptation penalty proportional to $\varepsilon^{-1 /(2 \gamma+1)}$. Furthermore, these results show that one cannot "adapt to the self-similarity 
constant:" if we require coverage for $\varepsilon$-self-similarity, then the adaptation penalty is proportional to $\varepsilon^{-1 /(2 \gamma+1)}$, even for functions that are $\varepsilon^{\prime}$-self-similar with $\varepsilon^{\prime}>\varepsilon$.

\subsection{Achieving the bound}

We now turn to upper bounds. Both of these bounds can be achieved simultaneously for all $\gamma \in[\underline{\gamma}, \bar{\gamma}]$ and $B \in[\underline{B}, \bar{B}]$ by a single confidence band, up to an additional term that depends only on $K$ and the range $[\gamma, \bar{\gamma}]$. We first state the upper bound, and then describe the confidence band that achieves it.

We make some additional assumptions on the kernel:

$$
\begin{aligned}
& \sup _{t \in[0,1]} \int K(t, x)^{2} d x<\infty \text { and there exists } \tau_{K}>0 \text { such that } \\
& \sup _{s, t \in[0,1]} \frac{\int[K(s, x)-K(t, x)]^{2} d x}{|s-t|^{\tau} K}<\infty .
\end{aligned}
$$

Condition (5) is a mild continuity condition. For convolution kernels $K(y, x)=\tilde{K}(y-x)$ or wavelet projection kernels $K(y, x)=\sum_{k} \phi(y-k) \phi(x-k)$, it is sufficient for the kernel $\tilde{K}$ or father wavelet $\phi$ to be bounded with finite support and bounded first derivative (see [12], p. 1146 for the latter case).

Theorem 2.3. Let $0<\underline{B}<\bar{B}$ and $0<\underline{\gamma}<\bar{\gamma}$ be given, and let $K$ be a kernel that satisfies (4) and (5), such that, for some $\tilde{C},(2)$ holds for all $B \in[\underline{B}, \bar{B}]$ and all $\gamma \in[\gamma, \bar{\gamma}]$. There exists a confidence band $\mathcal{C}_{n}(\cdot)$ and a constant $C_{K, \bar{\gamma}, \tilde{C}}^{*}$ depending only on $K, \bar{\gamma}$ and $\tilde{C}$ such that, with probability approaching one uniformly over $\bigcup_{\gamma \in[\underline{\gamma}, \bar{\gamma}]} \bigcup_{B \in[\underline{B}, \bar{B}]} \mathcal{F}_{\text {self-sim }}(\gamma, B, \varepsilon)$,

$$
\sup _{x \in[0,1]} \operatorname{length}\left(\mathcal{C}_{n}(x)\right) \leq C_{K, \bar{\gamma}, \tilde{C}}^{*}\left(B \varepsilon^{-1}\right)^{1 /(2 \gamma+1)}\left(\sigma_{n}^{2} \log \left(1 / \sigma_{n}^{2}\right)\right)^{\gamma /(2 \gamma+1)}
$$

and $f(x) \in \mathcal{C}_{n}(x)$ all $x \in[0,1]$.

To prove this theorem, we construct a confidence band that has coverage for the class $\bigcup_{B \in[\underline{B}, \bar{B}]} \bigcup_{\gamma \in[\gamma, \bar{\gamma}]} \mathcal{F}_{\mathrm{GN}}(\gamma, \varepsilon B, B)$, such that the width is bounded by a constant times $\left(\varepsilon^{-1} B\right)^{1 /(2 \gamma+1)}\left(\sigma_{n} \log \left(1 / \sigma_{n}\right)\right)^{\gamma /(2 \gamma+1)}$ with probability approaching one uniformly over the class $\mathcal{F}_{\mathrm{GN}}(\gamma, \varepsilon B, B)$. Letting $\tilde{\varepsilon}=\varepsilon / \tilde{C}$ and $\tilde{B}=\tilde{C} B$, we have $\mathcal{F}_{\text {self-sim }}(\varepsilon, \gamma, B) \subseteq \mathcal{F}_{\mathrm{GN}}(\gamma, \tilde{\varepsilon} \tilde{B}, \tilde{B})$ under (2), so that the conclusion of Theorem 2.3 holds for this confidence band, constructed with $\tilde{\varepsilon}=\varepsilon / \tilde{C}$ in place of $\varepsilon$. We describe the confidence band here, with additional details in the Appendix.

Let $\Delta\left(j, j^{\prime} ; f\right)=\sup _{x \in[0,1]}\left|K_{j} f(x)-K_{j^{\prime}} f(x)\right|$ and $\hat{\Delta}\left(j, j^{\prime}\right)=\sup _{x \in[0,1]}\left|\hat{f}(x, j)-\hat{f}\left(x, j^{\prime}\right)\right|$. Let $c(j)$ and $\tilde{c}\left(j, j^{\prime}\right)$ be critical values satisfying

$$
\left|\hat{f}(x, j)-K_{j} f(x)\right| \leq c(j) \quad \text { all } x \in[0,1], j \in \mathcal{J}_{n}
$$

and

$$
\left|\hat{\Delta}\left(j, j^{\prime}\right)-\Delta\left(j, j^{\prime} ; f\right)\right| \leq \tilde{c}\left(j, j^{\prime}\right) \quad \text { all } j, j^{\prime} \in \mathcal{J}_{n}
$$

with some prespecified probability for all $f \in \bigcup_{\gamma \in[\underline{\gamma}, \bar{\gamma}]} \bigcup_{B \in[\underline{B}, \bar{B}]} \mathcal{F}_{\mathrm{GN}}(\gamma, \varepsilon B, B)$, where $\mathcal{J}_{n}=$ $\left\{\underline{\ell}_{n}, \underline{\ell}_{n}+1, \ldots, \bar{\ell}_{n}\right\}$ for some $\underline{\ell}_{n}, \bar{\ell}_{n}$ (it suffices to set $c(j)=\bar{c}_{K} \sigma_{n} 2^{j / 2} \sqrt{j}$ and $\tilde{c}\left(j, j^{\prime}\right)=c(j)+c\left(j^{\prime}\right)$ for a large enough constant $\bar{c}_{K}$ and to take $\underline{\ell}_{n} \rightarrow \infty$ with $\underline{\ell}_{n} / \log n \rightarrow 0$ and $\bar{\ell}_{n} / \log n \rightarrow \infty$; see the Appendix). We construct a confidence band that covers $f$ for all $f \in \bigcup_{\gamma \in[\underline{\gamma}, \bar{\gamma}]} \bigcup_{B \in[\underline{B}, \bar{B}]} \mathcal{F}_{\mathrm{GN}}(\gamma, \varepsilon B, B$; $K, \underline{\ell}_{n}$ ) on the event that (6) and (7) both hold. 
To this end, we use $\Delta\left(j, j^{\prime} ; f\right)$ along with the self-similarity condition to bound the bias $\mid K_{j} f(x)-$ $f(x) \mid$. This, along with the confidence bands $\hat{f}(x, j) \pm c(j)$ and $\hat{\Delta}\left(j, j^{\prime}\right) \pm \tilde{c}\left(j, j^{\prime}\right)$ for $K_{j} f(x)$ and $\Delta\left(j, j^{\prime} ; f\right)$ leads to a confidence band for $f$. First, note that, for $f \in \mathcal{F}_{\mathrm{GN}}(\gamma, \varepsilon B, B ; K, \underline{\ell})$ and $j_{1}, j_{2} \geq$ $\underline{\ell}$

$$
\begin{aligned}
B\left(\varepsilon 2^{-j_{1} \gamma}-2^{-j_{2} \gamma}\right) & \leq \sup _{x \in[0,1]}\left|K_{j_{1}} f(x)-f(x)\right|-\sup _{x \in[0,1]}\left|K_{j_{2}} f(x)-f(x)\right| \\
& \leq \Delta\left(j_{1}, j_{2} ; f\right) \leq \sup _{x \in[0,1]}\left|K_{j_{1}} f(x)-f(x)\right|+\sup _{x \in[0,1]}\left|K_{j_{2}} f(x)-f(x)\right| \\
& \leq B\left(2^{-j_{1} \gamma}+2^{-j_{2} \gamma}\right),
\end{aligned}
$$

where the second and third inequalities are applications of the triangle inequality. For $0<\gamma_{\ell}<\gamma_{u}$, define

$$
a\left(\varepsilon, j_{1}, j_{2}, j, \gamma_{\ell}, \gamma_{u}\right)=\max \left\{\varepsilon 2^{-\max \left\{\left(j_{1}-j\right) \gamma_{u},\left(j_{1}-j\right) \gamma_{\ell}\right\}}-2^{-\min \left\{\left(j_{2}-j\right) \gamma_{u},\left(j_{2}-j\right) \gamma_{\ell}\right\}}, 0\right\}
$$

If $\gamma_{\ell} \leq \gamma \leq \gamma_{u}$ and $a\left(\varepsilon, j_{1}, j_{2}, j, \gamma_{\ell}, \gamma_{u}\right)>0$, then $a\left(\varepsilon, j_{1}, j_{2}, j, \gamma_{\ell}, \gamma_{u}\right) \leq \frac{\varepsilon 2^{-j_{1} \gamma}-2^{-j_{2} \gamma}}{2^{-j \gamma}}$ so that, for any $f \in \mathcal{F}_{\mathrm{GN}}(\gamma, \varepsilon B, B)$,

$$
\sup _{x \in[0,1]}\left|K_{j} f(x)-f(x)\right| \leq B 2^{-j \gamma} \leq B \frac{\varepsilon 2^{-j_{1} \gamma}-2^{-j_{2} \gamma}}{a\left(\varepsilon, j_{1}, j_{2}, j, \gamma_{\ell}, \gamma_{u}\right)} \leq \frac{\Delta\left(j_{1}, j_{2} ; f\right)}{a\left(\varepsilon, j_{1}, j_{2}, j, \gamma_{\ell}, \gamma_{u}\right)},
$$

where the last inequality uses (8).

In Appendix A.2, we provide an interval $\left[\hat{\gamma}_{\ell}, \hat{\gamma}_{u}\right]$ that contains $\gamma$ on the event in (7). Letting $\hat{\jmath}, \hat{J}_{1}$ and $\hat{\jmath}_{2}$ be data dependent values that are contained in $\mathcal{J}_{n}$ with probability one, it follows from (9) that, on the event that (6) and (7) both hold, the band

$$
\hat{f}(x, \hat{\jmath}) \pm\left[c(\hat{\jmath})+\frac{\hat{\Delta}\left(\hat{\jmath}_{1}, \hat{\jmath}_{2}\right)+\tilde{c}\left(\hat{\jmath}_{1}, \hat{\jmath}_{2}\right)}{a\left(\varepsilon, \hat{\jmath}_{1}, \hat{\jmath}_{2}, \hat{\jmath}, \hat{\gamma}_{\ell}, \hat{\gamma}_{u}\right)}\right]
$$

contains $f(x)$ for all $x \in[0,1]$. Since $\hat{\jmath}_{1}, \hat{\jmath}_{2}$ and $\hat{\jmath}$ can be data dependent, we can simply choose them to minimize the length of this band. For concreteness, we will assume that $\mathcal{J}_{n}$ is finite for each $n$, so that a minimum is taken:

$$
c(\hat{\jmath})+\frac{\hat{\Delta}\left(\hat{\jmath}_{1}, \hat{\jmath}_{2}\right)+\tilde{c}\left(\hat{\jmath}_{1}, \hat{\jmath}_{2}\right)}{a\left(\varepsilon, \hat{\jmath}_{1}, \hat{\jmath}_{2}, \hat{\jmath}, \hat{\gamma}_{\ell}, \hat{\gamma}_{u}\right)}=\min _{j, j_{1}, j_{2} \in \mathcal{J}_{n}}\left[c(j)+\frac{\hat{\Delta}\left(j_{1}, j_{2}\right)+\tilde{c}\left(j_{1}, j_{2}\right)}{a\left(\varepsilon, j_{1}, j_{2}, j, \hat{\gamma}_{\ell}, \hat{\gamma}_{u}\right)}\right],
$$

where we use the convention that $\frac{\hat{\Delta}\left(j_{1}, j_{2}\right)+\tilde{c}\left(j_{1}, j_{2}\right)}{a\left(\varepsilon, j_{1}, j_{2}, j, \hat{\gamma}_{\ell}, \hat{\gamma}_{u}\right)}$ is equal to $+\infty$ if $a\left(\varepsilon, j_{1}, j_{2}, j, \hat{\gamma}_{\ell}, \hat{\gamma}_{u}\right)=0$, so that the minimum is only over $j, j_{1}, j_{2}$ such that $a\left(\varepsilon, j_{1}, j_{2}, j, \hat{\gamma}_{\ell}, \hat{\gamma}_{u}\right)>0$. The half-length of this band is then bounded by

$$
\min _{j, j_{1}, j_{2} \in \mathcal{J}_{n}}\left[c(j)+\frac{B\left(2^{-j_{1} \gamma}+2^{-j_{2} \gamma}\right)+2 \tilde{c}\left(j_{1}, j_{2}\right)}{a\left(\varepsilon, j_{1}, j_{2}, j, \hat{\gamma}_{\ell}, \hat{\gamma}_{u}\right)}\right]
$$

on the event that (6) and (7) both hold (here we use the upper bound in (8)). In Appendix A.3, we use this bound to show that this confidence band, constructed with $\tilde{\varepsilon}=\varepsilon / \tilde{C}$ in place of $\varepsilon$, satisfies the requirements of Theorem 2.3 . 


\subsection{Alternative definition of self-similarity constant}

We have defined $\mathcal{F}_{\text {self-sim }}(\gamma, B, \varepsilon)$ to be the class of functions in $\mathcal{F}_{\text {Höl }}(\gamma, B)$ such that the lower bound in (3) holds with $b_{1}=\varepsilon B$. Under (2), this means that the self-similarity constant $\varepsilon$ gives the ratio between the upper and lower bound on bias, up to the constant $\tilde{C}$. The coverage condition takes the union of these classes with $\varepsilon$ fixed, so that large values of the Hölder constant require proportionally large values of the lower bound.

Alternatively, one could fix the lower bound $b_{1}=\varepsilon B$ when taking the union of these classes. This leads to the class $\overline{\mathcal{F}}_{\text {self-sim }}\left(\gamma, B, b_{1}\right)=\mathcal{F}_{\text {self-sim }}\left(\gamma, B, b_{1} / B\right)$. Of course, this does not change the conclusion of Theorem 2.2 (adaptation to $\gamma$ with $B$ fixed) since the formulation of this problem remains the same. For adaptation to $B$, however, we obtain a different formulation, with coverage required over the class $\bigcup_{B \in[\underline{B}, \bar{B}]} \overline{\mathcal{F}}_{\text {self-sim }}\left(\gamma, B, b_{1}\right)=\overline{\mathcal{F}}_{\text {self-sim }}\left(\gamma, \bar{B}, b_{1}\right)=\mathcal{F}_{\text {self-sim }}\left(\gamma, \bar{B}, b_{1} / \bar{B}\right)$. As the next theorem shows, this leads to a much more negative result: adaptation to the Hölder constant is completely impossible.

Theorem 2.4. Let $\gamma>0$ and let $0<2 \alpha<\beta<1$. Let $K$ be a kernel satisfying (4). There exists $\underline{j}_{K, \gamma}$, $C_{K, \gamma, *}>0$ and $\eta_{K, \gamma}>0$ such that, for any $0<B \leq \bar{B}, b_{1} \leq \eta_{K, \gamma} B$ and $\underline{\ell} \geq \underline{j}_{K, \gamma}$,

$$
\begin{aligned}
& R_{n, \alpha, \beta}^{*}\left(\overline{\mathcal{F}}_{\text {self-sim }}\left(\gamma, B, b_{1} ; K, \underline{\ell}\right), \overline{\mathcal{F}}_{\text {self-sim }}\left(\gamma, \bar{B}, b_{1} ; K, \underline{\ell}\right)\right) \\
& \quad \geq(1+o(1)) C_{K, \gamma, *} \bar{B}^{1 /(2 \gamma+1)}\left(\sigma_{n}^{2} \log \left(1 / \sigma_{n}\right)\right)^{\gamma /(2 \gamma+1)} .
\end{aligned}
$$

\subsection{Discussion}

The confidence band in Section 2.2 builds on the important work of [5] and [10] in constructing an upper bound on bias and using this to widen the confidence interval (see also [1,11,17] for confidence intervals for $f$ at a point in the nonadaptive case). In contrast to these papers, which derive bounds on the bias of an estimator with bandwidth selected using Lepski's method, we bound the bias directly for each bandwidth and use the width of the resulting confidence band to choose the bandwidth (note, however, that the two approaches are related, since the bound on the bias ultimately comes from comparisons of estimates at different bandwidths, either explicitly in our approach, or implicitly through the use of Lepski's method to choose the bandwidth). This makes it easier to derive explicit bounds, and it may be needed to get the optimal form $C \varepsilon^{-1 /(2 \gamma+1)}$ of the adaptation penalty ([5] and [10] show that their procedures are adaptive up to a constant, but do not derive how this constant depends on $\varepsilon$ ).

An alternative approach to ensuring coverage, used by [12], is undersmoothing, which uses a bandwidth sequence for which variance slightly dominates bias. As noted by [5] and [10], this leads to a slightly slower rate of convergence, so that the confidence band is not fully adaptive. Our lower bounds shed some light on this question: one must always pay an adaptation penalty of order $\varepsilon^{-1 /(2 \gamma+1)}$ when $\varepsilon$ is fixed, which means that letting $\varepsilon=\varepsilon_{n} \rightarrow 0$ requires paying a penalty in the rate. In practice, however, for any given finite sample size $n$, one only achieves coverage over a class $\mathcal{F}_{\text {self-sim }}$ corresponding to some $\varepsilon_{n}>0$; undersmoothed confidence bands choose such a sequence implicitly. To make this transparent, one can explicitly specify $\varepsilon_{n}$, and report a confidence band that is valid for the given selfsimilarity constant and noise level, even if the "asymptotic promise" states that $\varepsilon_{n} \rightarrow 0$ (while our arguments do not formally cover the case where $\varepsilon=\varepsilon_{n} \rightarrow 0$, it appears that they could be extended to allow $\varepsilon_{n} \rightarrow 0$ at a slow enough rate).

There has been some discussion in the literature of whether or how self-similarity conditions can lead to a practical approach to constructing confidence bands. If "practical" means that the confidence 
band should not require the user to choose any regularity constants a priori, then our results show that the answer is "no." On the other hand, if one sees the self-similarity constant as an interpretable object, then we need not be so pessimistic. Indeed, the confidence band we construct is "practical" in the sense that it has valid coverage for a given noise level without relying on conservative constants or sequences.

It is helpful to contrast the role of self-similarity conditions in our setting with regularity conditions used to construct confidence intervals for the mean of a univariate random variable. To form a nontrivial confidence interval for the mean of a univariate random variable, one must place some conditions on the tails of the distribution (see [2]). One approach is to choose some $\delta>0$, and assume that the $2+\delta$ moment is bounded by $1 / \delta$. Subject to this coverage requirement, the optimal width of the confidence interval does not depend on $\delta$ asymptotically: adding and subtracting the $1-\alpha / 2$ quantile of a normal distribution times the sample standard deviation leads to an asymptotically valid confidence interval regardless of the particular choice of $\delta>0$. Thus, one can state that this confidence interval is asymptotically valid and optimal under a bounded $2+\delta$ moment, without worrying about the exact choice of $\delta$. Our results show that this is not the case with self-similarity constants: no single confidence band is asymptotically valid and optimal under $\varepsilon$-self-similarity for all $\varepsilon$.

\section{Proofs of lower bounds}

This section proves Theorems 2.1, 2.2 and 2.4. To prove these lower bounds, we proceed as follows. Let $\widetilde{\mathcal{F}}(\gamma, B, a, b)$ denote the class of functions in $\mathcal{F}_{\text {Höl }}(\gamma, B)$ supported on $[a, b]$ :

$$
\widetilde{\mathcal{F}}(\gamma, B, a, b)=\left\{f \in \mathcal{F}_{\text {Höl }}(\gamma, B): f(t)=0 \text { all } t \notin[a, b]\right\} .
$$

While functions in $\widetilde{\mathcal{F}}(\gamma, B, a, b)$ need not be self-similar since this class does not impose a lower bound on bias, we can ensure self-similarity by adding a function supported outside of $[a, b]$ to this class, so long as this function satisfies the necessary upper and lower bounds (after adjusting some constants).

Section 3.1 presents a lower bound for adaptation to the singleton class $\{g\}$ for confidence bands with coverage under $g$ and under the class $\{f\}+\widetilde{\mathcal{F}}(\gamma, B, a, b)$, for any functions $f$ and $g$ supported outside of $[a, b]$. Following standard arguments relating adaptive confidence sets to minimax testing, such a bound follows so long as it is difficult to test between $f$ and $g$ (which holds if $f$ and $g$ are close in $L_{2}$ norm), by showing that it is difficult to test between $\{0\}$ (the zero function) and functions in $\widetilde{\mathcal{F}}(\gamma, B, a, b)$ for which the supremum over $[a, b]$ is sufficiently far from zero (which essentially follows from [19]). Section 3.2 constructs functions $g$ and $f$ such that the classes used in Section 3.1 satisfy the self-similarity condition for appropriate $B, \gamma$ and $\varepsilon$, so that the the lower bound in Section 3.1 can be used to give bounds on adaptation between self-similarity classes. For Theorems 2.1 and 2.4, the functions $g$ and $f$ can be taken to be equal, and the result follows almost immediately; Section 3.3 gives the necessary details to complete the proofs. To complete the proof of Theorem 2.2, we use the results in Section 3.2 to construct a function $g \in \mathcal{F}_{\text {self-sim }}\left(\gamma, 1, \varepsilon^{\prime}\right)$ and a sequence of functions $f_{n}$ converging to $g$ such that $\left\{f_{n}\right\}+\widetilde{\mathcal{F}}\left(\gamma-\delta_{n}, 1 / 2, a, b\right) \subseteq \mathcal{F}_{\text {self-sim }}\left(\gamma-\delta_{n}, 1, \varepsilon\right)$ where $\delta_{n}$ is a sequence converging to zero. Theorem 2.2 then follows by using the lower bounds in Section 3.1 and choosing the sequence $\delta_{n}$ to ensure that $f_{n}$ converges to $g$ quickly enough, while making the testing problem for the class $\widetilde{\mathcal{F}}\left(\gamma-\delta_{n}, 1 / 2, a, b\right)$ sufficiently difficult. These arguments are given in Section 3.4. 


\subsection{General lower bound}

In this section, we prove the following lower bound for adaptation between classes of the form $\{g\}+$ $\tilde{\mathcal{F}}(\gamma, B, a, b)$. For a function $f: \mathbb{R} \rightarrow \mathbb{R}$, let $\|f\|=\sqrt{\int f(t)^{2} d t}$ denote the $L_{2}$ norm of the function $f$.

Lemma 3.1. Let $a<b$ be given, and let $f_{n}$ and $g_{n}$ be sequences offunctions with $f_{n}(t)=g_{n}(t)=0$ for $t \in[a, b]$. Suppose $\left\|f_{n}-g_{n}\right\| / \sigma_{n} \rightarrow 0$. Let $0<\gamma \leq \bar{\gamma}$ be given, and let $\kappa$ be a function with finite support with $\kappa \in \mathcal{F}_{\mathrm{Höl}}(\gamma, 1)$ for all $\gamma \in(0, \bar{\gamma}]$. Let $B>0$ and let $C(\gamma, B, \kappa)=\left[\frac{4}{2 \gamma+1} B^{1 / \gamma} /\|\kappa\|^{2}\right]^{\frac{\gamma}{2 \gamma+1}} \kappa(0)$. Then, for any sequence $\gamma_{n} \in[\gamma, \bar{\gamma}]$ and any $0<2 \alpha<\beta<1$,

$$
\begin{aligned}
& R_{n, \alpha, \beta}^{*}\left(\left\{g_{n}\right\},\left\{\left\{f_{n}\right\}+\widetilde{\mathcal{F}}\left(\gamma_{n}, B, a, b\right)\right\} \cup\left\{g_{n}\right\}\right) \\
& \quad \geq C\left(\gamma_{n}, B, \kappa\right)\left(\sigma_{n}^{2} \log \left(1 / \sigma_{n}\right)\right)^{\gamma_{n} /\left(2 \gamma_{n}+1\right)}(1+o(1)) .
\end{aligned}
$$

To prove this result, we begin with a lemma relating $R_{n, \alpha, \beta}^{*}$ to minimax bounds on statistical hypothesis tests. For sets $\mathcal{F}$ and $\mathcal{G}$, let $d_{\text {test }}(\mathcal{F}, \mathcal{G})$ denote the maximum difference between minimax power and size of a test of $H_{0}: \mathcal{F}$ vs. $H_{1}: \mathcal{G}$ :

$$
d_{\text {test }}(\mathcal{F}, \mathcal{G})=\sup _{\phi} \inf _{f \in \mathcal{F}, g \in \mathcal{G}}\left|E_{g} \phi(Y)-E_{f} \phi(Y)\right|,
$$

where $E_{f}$ denotes expectation under the function $f$, and the supremum is over all tests $\phi$ based on $Y$ observed at noise level $\sigma_{n}$ (i.e., all measurable functions with range $[0,1]$ ). The following lemma is essentially Lemma 6.1 in [28], with the conclusion of the argument stated nonasymptotically.

Lemma 3.2. Let $\alpha, \beta$ and $\tilde{R}$ be given and let $\mathcal{G} \subseteq \mathcal{F}$. Suppose that

$$
\text { for some } f_{0} \in \mathcal{G}, \quad d_{\text {test }}\left(\left\{f_{0}\right\}, \mathcal{F} \cap\left\{f: \sup _{x \in[0,1]}\left|f(x)-f_{0}(x)\right| \geq \tilde{R}\right\}\right)<\beta-2 \alpha \text {. }
$$

Then $R_{n, \alpha, \beta}^{*}(\mathcal{G}, \mathcal{F}) \geq R_{n, \alpha, \beta}^{*}\left(\left\{f_{0}\right\}, \mathcal{F}\right) \geq \tilde{R}$.

Proof. Suppose, to get a contradiction, that $R_{n, \alpha, \beta}^{*}\left(\left\{f_{0}\right\}, \mathcal{F}\right)<\tilde{R}$. Then there exists a confidence band $\mathcal{C}_{n}(\cdot) \in \mathcal{I}_{n, \alpha, \mathcal{F}}$ with $R=R_{\beta}\left(\mathcal{C}_{n} ;\left\{f_{0}\right\}\right)=q_{\beta, f_{0}}\left(\sup _{x \in[0,1]} \operatorname{length}\left(\mathcal{C}_{n}(x)\right)\right)<\tilde{R}$, so that

$$
\begin{aligned}
& P_{f_{0}}\left(\sup _{x \in[0,1]} \text { length }\left(\mathcal{C}_{n}(x)\right)>R\right) \\
& \quad=1-P_{f_{0}}\left(\sup _{x \in[0,1]} \text { length }\left(\mathcal{C}_{n}(x)\right) \leq R\right) \leq 1-\beta .
\end{aligned}
$$

Let us abuse notation slightly and let $\mathcal{C}_{n}$ denote the set of functions $f$ contained in the confidence band $\mathcal{C}_{n}(\cdot)$, so that $f \in \mathcal{C}_{n}$ iff. $f(t) \in \mathcal{C}_{n}(t)$ all $t \in[0,1]$. Let $\phi=1$ if there exists a function $f$ satisfying $f \in \mathcal{F} \cap\left\{f: \sup _{x \in[0,1]}\left|f(x)-f_{0}(x)\right| \geq \tilde{R}\right\}$ with $f \in \mathcal{C}_{n}$. It is immediate from the definition of this test and the assumption that $\mathcal{C}_{n}(\cdot) \in \mathcal{I}_{n, \alpha, \mathcal{F}}$ that

$$
\inf _{f \in \mathcal{F} \cap\left\{f: \sup _{x \in[0,1]}\left|f(x)-f_{0}(x)\right| \geq \tilde{R}\right\}} E_{f} \phi \geq 1-\alpha
$$

(i.e., the test has minimax power at least $1-\alpha$ for $H_{1}: \mathcal{F} \cap\left\{f: \sup _{x \in[0,1]}\left|f(x)-f_{0}(x)\right| \geq \tilde{R}\right\}$ ). 
Now consider the level of the test for $H_{0}:\left\{f_{0}\right\}$. We have

$$
E_{f_{0}} \phi(Y)=E_{f_{0}} \phi(Y) I\left(f_{0} \in \mathcal{C}_{n}\right)+E_{f_{0}} \phi(Y) I\left(f_{0} \notin \mathcal{C}_{n}\right) \leq E_{f_{0}} \phi(Y) I\left(f_{0} \in \mathcal{C}_{n}\right)+\alpha
$$

by the converge condition. The event $\phi(Y) I\left(f_{0} \in \mathcal{C}_{n}\right)$ implies that $\mathcal{C}_{n}$ contains both $f_{0}$ and a function $f_{1}$ with $f_{1} \in \mathcal{F}$ and $\sup _{x \in[0,1]}\left|f_{1}(x)-f_{0}(x)\right| \geq \tilde{R}$. This, in turn, implies that $\sup _{x \in[0,1]}$ length $\left(\mathcal{C}_{n}(x)\right) \geq$ $\tilde{R}>R$ on this event so that, by (11), the probability of this event under $f_{0}$ is bounded by $1-\beta$. Thus, by the above display, $E_{f_{0}} \phi(Y) \leq 1-\beta+\alpha$. Combining this with (12), it follows that $\inf _{f \in \mathcal{F} \cap\left\{f: \sup _{x \in[0,1]}\left|f(x)-f_{0}(x)\right| \geq \tilde{R}\right\}} E_{f} \phi-E_{f_{0}} \phi \geq 1-\alpha-1+\beta-\alpha=\beta-2 \alpha$, which contradicts the assumptions of the theorem.

To deal with minimax tests over classes that add functions $f_{n}$ and $g_{n}$, we will also need the following lemma.

Lemma 3.3. For any functions $f_{0}$ and $g_{0}$ and sets $\mathcal{F}$ and $\mathcal{G}$,

$$
\begin{aligned}
& d_{\text {test }}\left(\mathcal{F}+\left\{f_{0}\right\}, \mathcal{G}+\left\{g_{0}\right\}\right) \\
& \quad=d_{\text {test }}\left(\mathcal{F}, \mathcal{G}+\left\{g_{0}-f_{0}\right\}\right) \\
& \leq d_{\text {test }}(\mathcal{F}, \mathcal{G})+\sup _{\alpha}\left[\Phi\left(\left\|f_{0}-g_{0}\right\| / \sigma_{n}-z_{1-\alpha}\right)-\alpha\right] \leq d_{\text {test }}(\mathcal{F}, \mathcal{G})+\left\|f_{0}-g_{0}\right\| / \sigma_{n} .
\end{aligned}
$$

Proof. The first equality follows since $f_{0}$ can be added or subtracted from $Y$ before performing any test, so that the supremum over tests $\phi(Y)$ is the same as the supremum over tests $\phi\left(Y-f_{0}\right)$. For the first inequality, note that

$$
\begin{aligned}
& d_{\text {test }}\left(\mathcal{F}, \mathcal{G}+\left\{g_{0}-f_{0}\right\}\right) \\
& \quad=\sup _{\phi} \inf _{f \in \mathcal{F}, g \in \mathcal{G}}\left|E_{g+f_{0}-g_{0}} \phi(Y)-E_{f} \phi(Y)\right| \\
& \quad \leq \sup _{\phi} \inf _{f \in \mathcal{F}, g \in \mathcal{G}}\left[\left|E_{g+f_{0}-g_{0}} \phi(Y)-E_{g} \phi(Y)\right|+\left|E_{g} \phi(Y)-E_{f} \phi(Y)\right|\right] .
\end{aligned}
$$

For any $g$, the first term is bounded by $\sup _{\phi}\left|E_{g+f_{0}-g_{0}} \phi(Y)-E_{g} \phi(Y)\right|$ which, using the NeymanPearson lemma and some calculations (see Example 2.1 in [15]), can be seen to be equal to

$$
\sup _{\alpha}\left[\Phi\left(\left\|f_{0}-g_{0}\right\| / \sigma_{n}-z_{1-\alpha}\right)-\Phi\left(z_{1-\alpha}\right)\right] \leq\left\|f_{0}-g_{0}\right\| / \sigma_{n}
$$

where the inequality follows from Taylor's theorem, since the derivative of the standard normal cdf is bounded by $1 / \sqrt{2 \pi} \leq 1$.

With these results in hand, we can now complete the proof of Lemma 3.1. Let $c_{n}=C\left(\gamma_{n}, B, \kappa\right) \times$ $\left(\sigma_{n}^{2} \log \left(1 / \sigma_{n}\right)\right)^{\gamma_{n} /\left(2 \gamma_{n}+1\right)}$. Given $\eta>0$, let

$$
\mathcal{H}_{n}=\left\{\left\{f_{n}\right\}+\widetilde{\mathcal{F}}\left(\gamma_{n}, B, a, b\right)\right\} \cap\left\{f: \sup _{x \in[0,1]}\left|f(x)-g_{n}(x)\right| \geq(1-\eta) c_{n}\right\} .
$$

By Lemma 3.2, the result will follow if we show that $d_{\text {test }}\left(\left\{g_{n}\right\}, \mathcal{H}_{n}\right) \rightarrow 0$. Furthermore, using the fact that $g_{n}$ and $f_{n}$ are supported outside $[a, b]$, it follows that $\left\{f_{n}\right\}+\widetilde{\mathcal{F}}\left(\gamma_{n}, B, a, b\right) \cap\{f$ : 
$\left.\sup _{x \in[a, b]}|f(x)| \geq(1-\eta) c_{n}\right\} \subseteq \mathcal{H}_{n}$. Since taking a smaller set increases $d_{\text {test }}$, it follows by Lemma 3.3, that $d_{\text {test }}\left(\left\{g_{n}\right\}, \mathcal{H}_{n}\right)$ is bounded by

$$
d_{\text {test }}\left(\{0\}, \widetilde{\mathcal{F}}\left(\gamma_{n}, B, a, b\right) \cap\left\{f: \sup _{x \in[a, b]}|f(x)| \geq(1-\eta) c_{n}\right\}\right)+\left\|f_{n}-g_{n}\right\| / \sigma_{n} .
$$

Since the second term converges to zero by assumption, it suffices to bound the first term.

To this end, we follow arguments on pp. 34-36 of [19]. Let $A_{\kappa}$ be a bound on the support of $\kappa$ and let

$$
\begin{aligned}
h_{n} & =\left(\frac{(1-\eta) C\left(\gamma_{n}, B, \kappa\right)}{B \kappa(0)}\right)^{1 / \gamma_{n}}\left(\sigma_{n}^{2} \log \left(1 / \sigma_{n}\right)\right)^{1 /\left(2 \gamma_{n}+1\right)}, \\
M_{n} & =\left\lfloor\frac{b-a}{2 A_{\kappa} h_{n}}\right\rfloor-1, \quad x_{n, k}=a+(2 k-1) A_{\kappa} h_{n}, \quad k=1, \ldots, M_{n} \\
f_{k, n}(x) & =B h_{n}^{\gamma_{n}} \kappa\left(\frac{x-x_{n, k}}{h_{n}}\right) .
\end{aligned}
$$

By construction, the support of each $f_{k, n}$ is nonoverlapping and contained in $[a, b]$. Also, the variance of $\int f_{k, n}(x) d Y(x)$ is

$$
B^{2} h_{n}^{2 \gamma_{n}} \int \kappa\left(\frac{x-x_{n, k}}{h_{n}}\right)^{2} d x=B^{2} h_{n}^{2 \gamma_{n}+1} \int \kappa(u)^{2} d u=: s_{n}^{2} .
$$

Following arguments on pp. 35-36 of [19], it will then follow that $d_{\text {test }}\left(\{0\},\left\{f_{n, 1}, f_{n, 2}, \ldots, f_{n, M_{n}}\right\}\right) \rightarrow$ 0 so long as there exists $\delta>0$ such that, for large enough $n,\left(s_{n}^{2} / \sigma_{n}^{2}\right) /\left(2 \log M_{n}\right) \leq(1-\delta)$. Since each $f_{k, n}$ is contained in the set $\widetilde{\mathcal{F}}\left(\gamma_{n}, B, a, b\right) \cap\left\{f: \sup _{x \in[a, b]}|f(x)|=(1-\eta) c_{n}\right\}$, this will complete the proof.

For large enough $n$, we have $M_{n} \geq(b-a) /\left(3 A_{\kappa} h_{n}\right)$ so that

$$
2 \log M_{n} \geq 2 \log h_{n}^{-1}+2 \log \left[(b-a) /\left(3 A_{\kappa}\right)\right]=\left(\frac{4}{2 \gamma_{n}+1}+o(1)\right) \log \left(1 / \sigma_{n}\right) .
$$

We have

$$
\begin{aligned}
\frac{s_{n}^{2}}{\sigma_{n}^{2}} & =B^{2}\|\kappa\|^{2} h_{n}^{2 \gamma_{n}+1} \sigma_{n}^{-2}=B^{2}\|\kappa\|^{2}\left(\frac{(1-\eta) C\left(\gamma_{n}, B, \kappa\right)}{B \kappa(0)}\right)^{\left(2 \gamma_{n}+1\right) / \gamma_{n}} \log \left(1 / \sigma_{n}\right) \\
& =(1-\eta)^{\left(2 \gamma_{n}+1\right) / \gamma_{n}} \frac{4}{2 \gamma_{n}+1} \log \left(1 / \sigma_{n}\right) .
\end{aligned}
$$

Thus, for $\delta$ smaller than a constant that depends only on $\bar{\gamma}$ and $\underline{\gamma}$, we have, for $n$ large enough, $\left(s_{n}^{2} / \sigma_{n}^{2}\right) /\left(2 \log M_{n}\right) \leq(1-\delta)$.

\subsection{Constructing functions in self-similarity classes}

The main result of this section is to construct functions $g$ such that the class $\{g\}+\widetilde{\mathcal{F}}(\gamma, B, a, b)$ satisfies the self-similarity condition. We first describe the construction, and then present the main lemma (Lemma 3.4) showing self-similarity of these functions. The remainder of this section is then devoted to the proof of Lemma 3.4. 
Let $\psi: \mathbb{R} \rightarrow \mathbb{R}$ be a function with $\|\psi\|=1$ with support contained in $\left(-C_{\psi}, C_{\psi}\right)$ where $C_{\psi}<\infty$. Let $\psi_{\ell k}(x)=2^{\ell / 2} \psi\left(2^{\ell} x-k\right)$. We will consider functions that take the form

$$
f_{\{\tilde{\beta}\}, \underline{\ell}}(x)=\sum_{\ell=\underline{\ell}}^{\infty} \tilde{\beta}_{\ell} \psi_{\ell k^{*}}(x),
$$

for integers $k^{*}, \underline{\ell}$, chosen large enough to satisfy conditions given below. Given $0<\varepsilon<1$ and $0<$ $\gamma-\delta \leq \gamma<\infty$, let $\tilde{f}_{\underline{\ell}, \gamma, \delta, \varepsilon, 1}$ be defined as in (13) with

$$
\tilde{\beta}_{\ell}=\max \left\{2^{-\ell(\gamma+1 / 2)}, \varepsilon 2^{-\ell(\gamma-\delta+1 / 2)}\right\} .
$$

Let $\tilde{g}_{\ell, \gamma, 1}$ be defined as in (13) with

$$
\tilde{\beta}_{\ell}=2^{-\ell(\gamma+1 / 2)}
$$

Let $\tilde{f}_{\underline{\ell}, \gamma, \delta, \varepsilon, A}(x)=A \tilde{f}_{\underline{\ell}, \gamma, \delta, \varepsilon, 1}(x)$ and let $\tilde{g}_{\underline{\ell}, \gamma, A}(x)=A \tilde{g}_{\underline{\ell}, \gamma, 1}(x)$.

To get some intuition for this construction, note that, if $\psi$ is a mother wavelet for some wavelet basis, then a function constructed in this way has $\ell, k$ th wavelet coefficient given by $\tilde{\beta}_{\ell}$ for $\ell \geq \underline{\ell}$ and $k=k^{*}$ and $\ell, k$ th wavelet coefficient 0 for all other $\ell, k$. If the kernel $K$ in the self-similarity condition is the wavelet projection kernel for this basis, self-similarity of $\tilde{g}_{\underline{\ell}, \gamma, A}$ and $\tilde{f}_{\underline{\ell}, \gamma, \delta, \varepsilon, A}$ would follow from standard calculations. However, relying on such arguments would rule out convolution kernels, and would also present an issue for nonsmooth wavelets (since we impose a Hölder condition in addition to the bounds on bias).

We now present the main result of this section, showing that, if $k^{*}$ and $\underline{\ell}$ are chosen appropriately, adding $\tilde{g}_{\underline{\ell}, \gamma, A}$ and $\tilde{f}_{\underline{\ell}, \gamma, \delta, \varepsilon, A}$ to functions in the classes $\widetilde{\mathcal{F}}(\gamma, B, a, b)$ yields self-similar functions. Let $\underline{C}_{K, \psi}=\sup _{x \in \mathbb{R}}\left|K_{0} \psi(x)-\psi(x)\right|>0$. Let $\|f\|_{\infty}=\sup _{t \in \mathbb{R}}|f(t)|$ denote the $L_{\infty}$ norm, and let $\bar{C}_{K, \psi, \gamma}=2\|\psi(\lfloor\gamma\rfloor+1)\|_{\infty}\left(2 C_{\psi}\right)^{1-(\gamma-\lfloor\gamma\rfloor)}$. Note that $\psi$ can be chosen so that $\bar{C}_{K, \psi, \gamma}$ is bounded from above over $\gamma \leq \bar{\gamma}$, and so that $\underline{C}_{K, \psi}>0$.

Lemma 3.4. Let $0<a<b, A>0$ and $\tilde{B} \geq 0$ be given, and let $K$ be a kernel that satisfies (4). Let $k^{*}>4\left(C_{\psi}+C_{K}\right)$, and let $\underline{\ell}$ be large enough so that $2^{-\underline{\ell}}\left(k^{*}+C_{\psi}+C_{K}\right)<a$. Then, for any $A^{*} \geq \bar{C}_{K, \psi, \gamma} A+\tilde{B}$ and $\varepsilon^{*} \leq \underline{C}_{K, \psi} A / A^{*}$,

$$
\tilde{\mathcal{F}}(\gamma, \tilde{B}, a, b)+\left\{\tilde{g}_{\underline{\ell}, \gamma, A}\right\} \subseteq \mathcal{F}_{\text {self-sim }}\left(\gamma, A^{*}, \varepsilon^{*} ; K, \underline{\ell}\right) .
$$

For any $A^{*} \geq \bar{C}_{K, \psi, \gamma-\delta} A+\tilde{B}, 0<\delta<\gamma$ and $\varepsilon^{*} \leq \tilde{\varepsilon} \underline{C}_{K, \psi} A / A^{*}$,

$$
\tilde{\mathcal{F}}(\gamma-\delta, \tilde{B}, a, b)+\left\{\tilde{f}_{\underline{\ell}, \gamma, \delta, \tilde{\varepsilon}, A}\right\} \subseteq \mathcal{F}_{\text {self-sim }}\left(\gamma-\delta, A^{*}, \varepsilon^{*} ; K, \underline{\ell}\right) .
$$

To prove Lemma 3.4, we first note some conditions on the support of the functions $\psi_{\ell k^{*}}$ and their projections.

Lemma 3.5. If the support of a function $f$ is contained in $(c, d)$ for some $c, d$, then the support of $K_{j} f$ is contained in $\left(c-2^{-j} C_{K}, d+2^{-j} C_{K}\right)$. In particular, letting $\tilde{S}_{j \ell}=\left(2^{-\ell} k^{*}-2^{-\ell} C_{\psi}-\right.$ $2^{-j} C_{K}, 2^{-\ell} k^{*}+2^{-\ell} C_{\psi}+2^{-j} C_{K}$ ) the support of $K_{j} \psi_{\ell k^{*}}$ is contained in $\tilde{S}_{j \ell}$, and the support of $\psi_{\ell k^{*}}$ is contained in $\tilde{S}_{\ell \ell}$. Furthermore, if $k^{*}>4\left(C_{\psi}+C_{K}\right)$, then $\tilde{S}_{j j} \cap \tilde{S}_{j \ell}=\varnothing$ for $\ell \neq j$. 
Proof. The first statement is immediate from the fact that $K_{j}(y, x)=2^{j} K\left(2^{j} y, 2^{j} x\right)=0$ whenever $|x-y|>2^{-j} C_{K}$. The second statement then follows since the support of $\psi_{\ell k^{*}}$ is contained in $\left(2^{-\ell} k^{*}-\right.$ $2^{-\ell} C_{\psi}, 2^{-\ell} k^{*}+2^{-\ell} C_{\psi}$ ) by the support condition on $\psi$. To verify the last statement, note that, for any $\ell \geq j+1$, elements in $\tilde{S}_{j \ell}$ are less than $2^{-j-1} k^{*}+2^{-j-1} C_{\psi}+2^{-j} C_{K}$, which is less than $2^{-j} k^{*}-$ $2^{-j} C_{\psi}-2^{-j} C_{K}$ (the lower support point of $\tilde{S}_{j j}$ ) so long as $k^{*}>3 C_{\psi}+4 C_{K}$, which is guaranteed by the condition $k^{*}>4\left(C_{\psi}+C_{K}\right)$. For any $\ell \leq j-1$, elements in $\tilde{S}_{j, \ell}$ are greater than $2^{-j+1} k^{*}-$ $2^{-j+1} C_{\psi}-2^{-j} C_{K}$, which is greater than $2^{-j} k^{*}+2^{-j} C_{\psi}+2^{-j} C_{K}$ (the upper support point of $\tilde{S}_{j j}$ ) so long as $k^{*}>3 C_{\psi}+2 C_{K}$, which is guaranteed by the condition $k^{*}>4\left(C_{\psi}+C_{K}\right)$.

We now use this to obtain a lower bound on projection bias.

Lemma 3.6. Suppose that $K(y, x)$ satisfies (4), and let $f_{\{\tilde{\beta}\}, \underline{\ell}}$ be defined as in (13), with $k^{*}>4\left(C_{\psi}+\right.$ $\left.C_{K}\right)$. Let $f^{*}$ be a function supported on the set $\left(2^{-\underline{\ell}}\left(k^{*}+C_{\psi}+2 C_{K}\right), \infty\right)$, and let $f=f_{\{\tilde{\beta}\}, \underline{\ell}}+f^{*}$. Then, for $j \geq \underline{\ell}$,

$$
\sup _{x \in\left[0,2^{-j}\left(k^{*}+C_{\psi}+C_{K}\right)\right]}\left|K_{j} f(x)-f(x)\right| \geq\left|\tilde{\beta}_{j}\right| \cdot 2^{j / 2} \sup _{x \in \mathbb{R}}\left|K_{0} \psi(x)-\psi(x)\right| .
$$

Proof. It follows from Lemma 3.5 that, for $x \in \tilde{S}_{j j}$, we have $f(x)=\psi_{j k^{*}}(x)$ and $K_{j} f(x)=$ $K_{j} \psi_{j k^{*}}(x)$, so that

$$
\begin{aligned}
& \sup _{x \in\left[0,2^{-j}\left(k^{*}+C_{\psi}+C_{K}\right)\right]}\left|K_{j} f(x)-f(x)\right| \\
& \geq \sup _{x \in \tilde{S}_{j j}}\left|K_{j} f(x)-f(x)\right| \\
& =\left|\tilde{\beta}_{j}\right| \sup _{x \in \mathbb{R}}\left|K_{j} \psi_{j k^{*}}(x)-\psi_{j k^{*}}(x)\right|=\left|\tilde{\beta}_{j}\right| \cdot 2^{j / 2} \sup _{x \in \mathbb{R}}\left|K_{0} \psi(x)-\psi(x)\right|,
\end{aligned}
$$

where the last step follows by using a change of variables to note that $K_{j} \psi_{j k^{*}}(x)-\psi_{j k^{*}}(x)=$ $2^{j / 2}\left[K_{0} \psi\left(u-k^{*}\right)-\psi\left(u-k^{*}\right)\right]$.

Next, we obtain a Hölder condition on functions of the form given in (13) using the rate of decay of the coefficients $\tilde{\beta}_{\ell}$.

Lemma 3.7. Let $\gamma>0$ and suppose that $\psi$ is $\lfloor\gamma\rfloor+1$ times differentiable. Let $A$ be given and let $f(x)=f_{\{\tilde{\beta}\}, \underline{\ell}}(x)$ be given by (13) where $\left|\tilde{\beta}_{\ell}\right| \leq A 2^{-\ell(\gamma+1 / 2)}$ for all $\ell$. Then $f \in \mathcal{F}_{\text {Höl }}(\gamma$, $\left.2 A\left\|\psi^{(\lfloor\gamma\rfloor+1)}\right\|_{\infty}\left(2 C_{\psi}\right)^{1-(\gamma-\lfloor\gamma\rfloor)}\right)$.

Proof. Since the supports of the functions $\psi_{\ell k^{*}}$ do not overlap with each other by Lemma 3.5, it follows from Lemma 3.8 below that it suffices to show that $x \mapsto \tilde{\beta}_{\ell} \psi_{\ell k^{*}}(x)$ is in $\mathcal{F}_{\text {Höl }}\left(\gamma, A\left\|\psi^{(\lfloor\gamma\rfloor)+1}\right\|_{\infty} \times\right.$ $\left.\left(2 C_{\psi}\right)^{1-(\gamma-\lfloor\gamma\rfloor)}\right)$ for each $\ell$. Given $\ell$, let $x$ and $x^{\prime}$ be in the support of $\psi_{\ell k^{*}}$ so that $x, x^{\prime} \in\left[2^{-\ell} k^{*}-\right.$ $\left.2^{-\ell} C_{\psi}, 2^{-\ell} k^{*}+2^{-\ell} C_{\psi}\right]$. Then

$$
\begin{aligned}
& \left|\tilde{\beta}_{\ell} \psi_{\ell k^{*}}^{(\lfloor\gamma\rfloor)}(x)-\tilde{\beta}_{\ell} \psi_{\ell k^{*}}^{(\lfloor\gamma\rfloor)}\left(x^{\prime}\right)\right| \\
& \quad=\left|\tilde{\beta}_{\ell}\right| 2^{\ell(\lfloor\gamma\rfloor+1 / 2)}\left|\psi^{(\lfloor\gamma\rfloor)}\left(2^{\ell} x+k\right)-\psi^{(\lfloor\gamma\rfloor)}\left(2^{\ell} x^{\prime}+k\right)\right| \\
& \quad \leq\left\|\psi^{(\lfloor\gamma\rfloor+1)}\right\|_{\infty} \cdot\left|\tilde{\beta}_{\ell}\right|^{\ell(\lfloor\gamma\rfloor+1 / 2)} \cdot 2^{\ell}\left|x-x^{\prime}\right|
\end{aligned}
$$




$$
\begin{aligned}
& =\left\|\psi^{(\lfloor\gamma\rfloor+1)}\right\|_{\infty} \cdot\left|\tilde{\beta}_{\ell}\right| 2^{\ell(\lfloor\gamma\rfloor+1 / 2)} \cdot\left(2 C_{\psi}\right) \cdot\left(2 C_{\psi}\right)^{-1} 2^{\ell}\left|x-x^{\prime}\right| \\
& \leq\left\|\psi^{(\lfloor\gamma\rfloor+1)}\right\|_{\infty} \cdot\left|\tilde{\beta}_{\ell}\right| 2^{\ell(\lfloor\gamma\rfloor+1 / 2)} \cdot\left(2 C_{\psi}\right) \cdot\left(2 C_{\psi}\right)^{-(\gamma-\lfloor\gamma\rfloor)} 2^{\ell(\gamma-\lfloor\gamma\rfloor)}\left|x-x^{\prime}\right|^{\gamma-\lfloor\gamma\rfloor},
\end{aligned}
$$

where the last inequality uses the fact that $\left(2 C_{\psi}\right)^{-1} 2^{\ell}\left|x-x^{\prime}\right| \leq 1$ by the conditions on $x, x^{\prime}$. If $\left|\tilde{\beta}_{\ell}\right| \leq$ $A 2^{-\ell(\gamma+1 / 2)}$, then this is bounded by $A\left\|\psi^{(\lfloor\gamma\rfloor+1)}\right\|_{\infty}\left(2 C_{\psi}\right)^{1-(\gamma-\lfloor\gamma\rfloor)}\left|x-x^{\prime}\right|^{\gamma-\lfloor\gamma\rfloor}$ as required.

We have used the following lemma.

Lemma 3.8. Let $\left\{g_{k}\right\}_{k=1}^{\infty}$ be a sequence of functions with nonoveralapping support with $g_{k} \in$ $\mathcal{F}_{\text {Höl }}(\gamma, B)$ for each $k$. Let $f=\sum_{k=1}^{\infty} g_{k}$. Then $f \in \mathcal{F}_{\text {Höl }}(\gamma, 2 B)$.

Proof. Let $x, x^{\prime}$ be given. We need to show that $\left|f^{\lfloor\gamma\rfloor}(x)-f^{\lfloor\gamma\rfloor}\left(x^{\prime}\right)\right| \leq 2 B\left|x-x^{\prime}\right|^{\gamma-\lfloor\gamma\rfloor}$. If $x$ and $x^{\prime}$ are both in the support of $g_{k}$ for some $k$, or if $x$ and $x^{\prime}$ are not in the support of $g_{k}$ for any $k$, then this follows immediately. If $x$ is in the support of $g_{k}$ and $x^{\prime}$ is in the support of $g_{k^{\prime}}$ for some $k^{\prime} \neq k$, let $\bar{x}$ denote the upper endpoint of the support of $g_{k}$ and let $\underline{x}^{\prime}$ denote the lower endpoint of the support of $g_{k^{\prime}}$, and assume without loss of generality that $\bar{x} \leq \underline{x}^{\prime}$. By the Hölder condition on $g_{k}$ and $g_{k^{\prime}}$, we have $g_{k}^{\lfloor\gamma\rfloor}(\bar{x})=g_{k^{\prime}}^{\lfloor\gamma\rfloor}\left(\underline{x}^{\prime}\right)=0$, so that $\left|f^{\lfloor\gamma\rfloor}(x)-f^{\lfloor\gamma\rfloor}\left(x^{\prime}\right)\right|=\mid g_{k}^{\lfloor\gamma\rfloor}(x)-g_{k}^{\lfloor\gamma\rfloor}(\bar{x})+g_{k^{\prime}}^{\lfloor\gamma\rfloor}(x)-$ $g_{k^{\prime}}^{\lfloor\gamma\rfloor}\left(\underline{x}^{\prime}\right)|\leq B| x-\left.\bar{x}\right|^{\gamma-\lfloor\gamma\rfloor}+B\left|x^{\prime}-\underline{x}^{\prime}\right|^{\gamma-\lfloor\gamma\rfloor} \leq 2 B\left|x-x^{\prime}\right|^{\gamma-\lfloor\gamma\rfloor}$. Finally, if $x$ is in the support of some $g_{k}$ and $x^{\prime}$ is not in the support of $g_{k^{\prime}}$ for any $k^{\prime}$, then, letting $[\underline{x}, \bar{x}]$ denote the support of $g_{k}$, $\left|f^{\lfloor\gamma\rfloor}(x)-f^{\lfloor\gamma\rfloor}\left(x^{\prime}\right)\right|=\left|g_{k}^{\lfloor\gamma\rfloor}(x)\right| \leq B \min \left\{|x-\underline{x}|^{\gamma-\lfloor\gamma\rfloor},|x-\bar{x}|^{\gamma-\lfloor\gamma\rfloor}\right\} \leq B\left|x-x^{\prime}\right|^{\gamma-\lfloor\gamma\rfloor}$.

With these results in hand, we can now prove Lemma 3.4. Let $f^{*} \in \tilde{\mathcal{F}}(\gamma, \tilde{B}, a, b)$ and let $g=\tilde{g}_{\underline{\ell}, \gamma, A}+f^{*}$ and $f=\tilde{f}_{\underline{\ell}, \gamma, \delta, \tilde{\varepsilon}, A}+f^{*}$. It follows from Lemma 3.7 that $\tilde{g}_{\underline{\ell}, \gamma, A} \in \mathcal{F}_{\text {Höl }}\left(\gamma, \bar{C}_{K, \psi, \gamma} A\right)$ and $\tilde{f}_{\ell, \gamma, \delta, \tilde{\varepsilon}, A} \in \mathcal{F}_{\text {Höl }}\left(\gamma-\delta, \bar{C}_{K, \psi, \gamma-\delta} A\right)$. Thus, $g \in \mathcal{F}_{\text {Höl }}\left(\gamma, \bar{C}_{K, \psi, \gamma} A+\tilde{B}\right) \subseteq \mathcal{F}_{\text {Höl }}\left(\gamma, A^{*}\right)$ for $A^{*} \geq$ $\bar{C}_{K, \psi, \gamma} A+\tilde{B}$ and $f \in \mathcal{F}_{\mathrm{Höl}}\left(\gamma-\delta, \bar{C}_{K, \psi, \gamma-\delta} A+\tilde{B}\right) \subseteq \mathcal{F}_{\mathrm{Höl}}\left(\gamma-\delta, A^{*}\right)$ for $A^{*} \geq \bar{C}_{K, \psi, \gamma-\delta} A+\tilde{B}$. To verify the lower bound on bias, note that, for $j \geq \underline{\ell}$, we have, by Lemma 3.6, $\sup _{x \in[0,1]} \mid K_{j} g(x)-$ $g(x) \mid \geq A 2^{-j(\gamma+1 / 2)} \cdot 2^{j / 2} \underline{C}_{K, \psi}=A 2^{-j \gamma} \underline{C}_{K, \psi}=\left(\underline{C}_{K, \psi} A / A^{*}\right) \cdot A^{*} \cdot 2^{-j \gamma}$. Thus, for $A^{*} \geq \bar{C}_{K, \psi, \gamma} A+\tilde{B}$ and $\varepsilon^{*} \leq \underline{C}_{K, \psi} A / A^{*}$, we have $g \in \mathcal{F}_{\text {self-sim }}\left(\gamma, A^{*}, \varepsilon^{*} ; K, \underline{\ell}\right)$ as required. Similarly, $\sup _{x \in[0,1]}\left|K_{j} f(x)-f(x)\right| \geq \tilde{\varepsilon} A 2^{-j(\gamma-\delta+1 / 2)} \cdot 2^{j / 2} \underline{C}_{K, \psi}=\tilde{\varepsilon} A 2^{-j(\gamma-\delta)} \underline{C}_{K, \psi}=\tilde{\varepsilon}\left(\underline{C}_{K, \psi} A / A^{*}\right) \cdot A^{*}$. $2^{-j(\gamma-\delta)}$, so that, for $A^{*} \geq \bar{C}_{K, \psi, \gamma-\delta} A+\tilde{B}$ and $\varepsilon^{*} \leq \tilde{\varepsilon} \underline{C}_{K, \psi} A / A^{*}$, we have $f \in \mathcal{F}_{\text {self-sim }}(\gamma-$ $\left.\delta, A^{*}, \varepsilon^{*} ; K, \underline{\ell}\right)$ as required.

\subsection{Proofs of Theorems 2.1 and 2.4}

To prove Theorem 2.1, let $\tilde{g}_{\underline{\ell}, \gamma, A}$ be defined as in Section 3.2 with $k^{*}$ and $\underline{\ell}$ chosen so that $k^{*}>4\left(C_{\psi}+\right.$ $\left.C_{K}\right)$ and $2^{-\ell}\left(k^{*}+C_{\psi}+C_{K}\right)<1 / 2$, and with $A=B /\left(2 \max \left\{\bar{C}_{K, \psi, \gamma}, 1\right\}\right)$. By Lemma 3.4, $\tilde{g}_{\ell, \gamma, A} \in$ $\mathcal{F}_{\text {self-sim }}\left(\gamma, B, \varepsilon^{\prime} ; K, \underline{\ell}\right)$ so long as $\varepsilon^{\prime} \leq \underline{C}_{K, \psi} /\left(2 \max \left\{\bar{C}_{K, \psi, \gamma}, 1\right\}\right)$. Let $\tilde{B}=\min \left\{\tilde{\varepsilon}^{-1} B, \bar{B}\right\}-\bar{C}_{K, \psi, \gamma} A$ where $\tilde{\varepsilon}=2 \varepsilon \max \left\{\bar{C}_{K, \psi, \gamma}, 1\right\} / \underline{C}_{K, \psi}$. Applying Lemma 3.4 with $\min \left\{\tilde{\varepsilon}^{-1} B, \bar{B}\right\}$ playing the role of $A^{*}$, we have $\widetilde{\mathcal{F}}(\gamma, \tilde{B}, 1 / 2,1)+\left\{\tilde{g}_{\underline{\ell}, \gamma, A}\right\} \subseteq \mathcal{F}_{\text {self-sim }}\left(\gamma, \min \left\{\tilde{\varepsilon}^{-1} B, \bar{B}\right\}, \varepsilon ; K, \underline{\ell}\right)$, where we use the fact that the choice of $\tilde{\varepsilon}$ guarantees $\underline{C}_{K, \psi} A / A^{*} \geq \varepsilon$. If $\eta_{K, \gamma}$ is small enough, then we will have $\min \left\{\tilde{\varepsilon}^{-1} B, \bar{B}\right\} \in$ $[\underline{B}, \bar{B}]$, so that this implies $\widetilde{\mathcal{F}}(\gamma, \tilde{B}, 1 / 2,1)+\left\{\tilde{g}_{\ell, \gamma, A}\right\} \subseteq \bigcup_{B^{\prime} \in[\underline{B}, \bar{B}]} \mathcal{F}_{\text {self-sim }}\left(\gamma, B^{\prime}, \varepsilon ; K, \underline{\ell}\right)$. Applying Lemma 3.1, it follows that $R_{n, \alpha, \beta}^{*}\left(\mathcal{F}_{\text {self-sim }}\left(\gamma, B, \varepsilon^{\prime} ; K, \underline{\ell}\right), \bigcup_{B^{\prime} \in[\underline{B}, \bar{B}]} \mathcal{F}_{\text {self-sim }}\left(\gamma, B^{\prime}, \varepsilon ; K, \underline{\ell}\right)\right)$ is bounded from below by $(1+o(1)) \tilde{B}^{1 /(2 \gamma+1)}\left(\sigma_{n}^{2} \log \left(1 / \sigma_{n}\right)\right)^{\gamma /(2 \gamma+1)}$ times a term that depends only on 
$\gamma$. The result follows by noting that, if $\eta_{K, \gamma}$ is chosen small enough, then $\tilde{B}$ is bounded from below by a constant times $\min \left\{\varepsilon^{-1} B, \bar{B}\right\}$, where the constant depends only on $\underline{C}_{K, \psi}$ and $\bar{C}_{K, \psi, \gamma}$.

To prove Theorem 2.4, we use similar arguments with the same function $\tilde{g}_{\ell, \gamma, A}$ (defined with $k^{*}$ and $\underline{\ell}$ chosen so that $k^{*}>4\left(C_{\psi}+C_{K}\right)$ and $2^{-\underline{\ell}}\left(k^{*}+C_{\psi}+C_{K}\right)<1 / 2$, and with $A=$ $\left.B /\left(2 \max \left\{\bar{C}_{K, \psi, \gamma}, 1\right\}\right)\right)$. By Lemma $3.4, \tilde{g}_{\ell, \gamma, A} \in \mathcal{F}_{\text {self-sim }}\left(\gamma, B, b_{1} / B ; K, \underline{\ell}\right)=\overline{\mathcal{F}}_{\text {self-sim }}\left(\gamma, B, b_{1}\right.$; $K, \underline{\ell})$ so long as $b_{1} / B \leq \underline{C}_{K, \psi} /\left(2 \max \left\{\bar{C}_{K, \psi, \gamma}, 1\right\}\right)$. Let $\tilde{B}=\bar{B}-\bar{C}_{K, \psi, \gamma} A=\bar{B}-B \bar{C}_{K, \psi, \gamma} /$ $\left(2 \max \left\{\bar{C}_{K, \psi, \gamma}, 1\right\}\right)$. Applying Lemma 3.4 with $\bar{B}$ playing the role of $A^{*}$, we have $\tilde{\mathcal{F}}(\gamma, \tilde{B}, 1 / 2,1)+$ $\left\{\tilde{g}_{\ell}, \gamma, A\right\} \subseteq \mathcal{F}_{\text {self-sim }}\left(\gamma, \bar{B}, b_{1} / \bar{B} ; K, \underline{\ell}\right)=\overline{\mathcal{F}}_{\text {self-sim }}\left(\gamma, \bar{B}, b_{1} ; K, \underline{\ell}\right)$, so long as $b_{1} \leq \underline{C}_{K, \psi} A=\underline{C}_{K, \psi} B /$ (2 $\left.\max \left\{\bar{C}_{K, \psi, \gamma}, 1\right\}\right)$. The result follows by applying Lemma 3.1 and noting that $\tilde{B} \geq \bar{B} / 2$.

\subsection{Proof of Theorem 2.2}

To prove Theorem 2.2, let $\bar{C}=\sup _{\gamma^{\prime} \in(0, \bar{\gamma}]} \bar{C}_{K, \psi, \gamma^{\prime}}$ and let $A=1 /(2 \bar{C})$ and $\tilde{\varepsilon}=2 \varepsilon \bar{C} / \underline{C}_{K, \psi}$. Let $\tilde{g}_{\underline{\ell}, \gamma, A}$ and $\tilde{f}_{\underline{\ell}, \gamma, \delta, \tilde{\varepsilon}, A}$ be defined as in Section 3.2 with $k^{*}$ and $\underline{\ell}$ chosen so that $k^{*}>4\left(C_{\psi}+C_{K}\right)$ and $2^{-\underline{\ell}}\left(k^{*}+C_{\psi}+C_{K}\right)<1 / 2$. By Lemma 3.4, we have $\tilde{g}_{\underline{\ell}, \gamma, A} \in \mathcal{F}_{\text {self-sim }}\left(\gamma, 1, \varepsilon^{\prime}\right) \subseteq \mathcal{F}_{\text {self-sim }}(\gamma, 1, \varepsilon)$ for any $\varepsilon \leq \varepsilon^{\prime} \leq \underline{C}_{K, \psi} /(2 \bar{C})$ and $\tilde{\mathcal{F}}(\gamma-\delta, 1 / 2,1 / 2,1)+\left\{\tilde{f}_{\underline{\ell}, \gamma, \delta, \tilde{\varepsilon}, A}\right\} \subseteq \mathcal{F}_{\text {self-sim }}(\gamma-\delta, 1, \varepsilon)$. Thus, applying Lemma 3.1, we have, for any positive sequence $\delta_{n} \rightarrow 0$,

$$
\begin{aligned}
& R_{n, \alpha, \beta}^{*}\left(\mathcal{F}_{\text {self-sim }}\left(\gamma, 1, \varepsilon^{\prime}\right), \bigcup_{\gamma^{\prime} \in[\underline{\gamma}, \bar{\gamma}]} \mathcal{F}_{\text {self-sim }}\left(\gamma^{\prime}, 1, \varepsilon\right)\right) \\
& \quad \geq C\left(\gamma-\delta_{n}, 1 / 2, \kappa\right)\left(\sigma_{n}^{2} \log \left(1 / \sigma_{n}\right)\right)^{\left(\gamma-\delta_{n}\right) /\left(2\left(\gamma-\delta_{n}\right)+1\right)}(1+o(1))
\end{aligned}
$$

so long as

$$
\left\|\tilde{g}_{\underline{\ell}, \gamma, A}-\tilde{f}_{\underline{\ell}, \gamma, \delta_{n}, \tilde{\varepsilon}, A}\right\| / \sigma_{n} \rightarrow 0 .
$$

Since $C\left(\gamma-\delta_{n}, 1 / 2, \kappa\right)$ is bounded from below by a positive constant that depends only on $\bar{\gamma}$, it suffices to find a sequence $\delta_{n} \rightarrow 0$ such that (14) holds and

$$
\liminf _{n \rightarrow \infty} \frac{\left(\sigma_{n}^{2} \log \left(1 / \sigma_{n}\right)\right)^{\left(\gamma-\delta_{n}\right) /\left(2\left(\gamma-\delta_{n}\right)+1\right)}}{\left(\sigma_{n}^{2} \log \left(1 / \sigma_{n}\right)\right)^{\gamma /(2 \gamma+1)}} \geq c \cdot \varepsilon^{-1 /(2 \gamma+1)}
$$

for some constant $c$ that depends only on $\bar{\gamma}$ and $K$.

Let $\delta_{n}=C_{n} / \log n$ where $C_{n}=\left(1-b_{n}\right)(2 \gamma+1) \log \tilde{\varepsilon}^{-1}$ with $b_{n}=1 /(\log n)^{1 / 2}$. First, note that $\left\|\tilde{g}_{\underline{\ell}, \gamma, A}-\tilde{f}_{\underline{\ell}, \gamma, \delta, \tilde{\varepsilon}, A}\right\|^{2}$ is equal to $A^{2}$ times

$$
\sum_{\ell=\tilde{\ell}}^{\infty}\left(\tilde{\varepsilon} 2^{-\ell(\gamma-\delta+1 / 2)}-2^{-\ell(\gamma+1 / 2)}\right)^{2}=\sum_{\ell=\tilde{\ell}}^{\infty} 2^{-\ell(2 \gamma+1)}\left(\tilde{\varepsilon} 2^{\ell \delta}-1\right)^{2},
$$

where $\tilde{\ell}=\tilde{\ell}(\tilde{\varepsilon}, \delta)$ is the minimum value of $\ell \geq \underline{\ell}$ such that $\tilde{\varepsilon} 2^{\ell \delta}>1$ (here we use the fact that the support of $\psi_{\ell k^{*}}$ does not overlap with the support of $\psi_{\ell^{\prime} k^{*}}$ for $\ell \neq \ell^{\prime}$ by Lemma 3.5). The above display is bounded by

$$
\tilde{\varepsilon}^{2} \sum_{\ell=\tilde{\ell}}^{\infty} 2^{-\ell(2(\gamma-\delta)+1)}=\tilde{\varepsilon}^{2} \sum_{\ell=0}^{\infty} 2^{-(\ell+\tilde{\ell})(2(\gamma-\delta)+1)}=\tilde{\varepsilon}^{2} 2^{-\tilde{\ell}(2(\gamma-\delta)+1)} \sum_{\ell=0}^{\infty} 2^{-\ell(2(\gamma-\delta)+1)} .
$$


Note that $2^{-\tilde{\ell}}<\tilde{\varepsilon}^{1 / \delta}$, so $2^{-\tilde{\ell}(2(\gamma-\delta)+1)}<\tilde{\varepsilon}^{(2(\gamma-\delta)+1) / \delta}$. From this and the fact that $\sum_{\ell=0}^{\infty} 2^{-\ell(2(\gamma-\delta)+1)} \leq \sum_{\ell=0}^{\infty} 2^{-\ell}=2$, it follows that the above display is bounded by $2 \tilde{\varepsilon}^{2+(2(\gamma-\delta)+1) / \delta}=2 \tilde{\varepsilon}^{(2 \gamma+1) / \delta}$. Plugging in $\delta_{n}=C_{n} / \log n$, dividing by $\sigma_{n}^{2}$ and taking logs gives

$$
\begin{aligned}
\log & {\left[\left\|\tilde{f}_{\underline{\ell}, \gamma, \delta_{n}, \tilde{\varepsilon}, A}-\tilde{g}_{\underline{\ell}, \gamma, A}\right\|^{2} / \sigma_{n}^{2}\right] } \\
& \leq \frac{2 \gamma+1}{\delta_{n}} \log \tilde{\varepsilon}+\log 2-\log \left(\sigma^{2} / n\right)+\log A^{2} \\
& =\left(\frac{(2 \gamma+1) \log \tilde{\varepsilon}}{C_{n}}+1\right) \log n+\log \left(2 A^{2} / \sigma^{2}\right)=\frac{-b_{n}}{1-b_{n}} \log n+\log \left(2 A^{2} / \sigma^{2}\right),
\end{aligned}
$$

which diverges to $-\infty$, so that exponentiating gives a sequence that converges to 0 . Thus, (14) holds for this sequence $\delta_{n}$.

To verify (15) for this sequence $\delta_{n}$, note that

$$
\frac{\gamma-\delta_{n}}{2\left(\gamma-\delta_{n}\right)+1}-\frac{\gamma}{2 \gamma+1}=-\frac{\delta_{n}}{\left[2\left(\gamma-\delta_{n}\right)+1\right](2 \gamma+1)}=-\frac{\delta_{n}}{(2 \gamma+1)^{2}}(1+o(1)) .
$$

Thus,

$$
\begin{aligned}
\left(\sigma_{n}^{2}\right)^{\frac{\gamma-\delta_{n}}{2\left(\gamma-\delta_{n}\right)+1}-\frac{\gamma}{2 \gamma+1}} & =\left(\sigma_{n}^{2}\right)^{-\frac{\delta_{n}}{(2 \gamma+1)^{2}}(1+o(1))}=(1+o(1)) n^{\frac{\delta_{n}}{(2 \gamma+1)^{2}}(1+o(1))} \\
& =\exp \left(\frac{\delta_{n}}{(2 \gamma+1)^{2}}(1+o(1)) \log n\right)
\end{aligned}
$$

Since $\delta_{n} \log n \rightarrow(2 \gamma+1) \log \tilde{\varepsilon}^{-1}$, this converges to $\exp \left(\frac{(2 \gamma+1) \log \tilde{\varepsilon}^{-1}}{(2 \gamma+1)^{2}}\right)=\tilde{\varepsilon}^{-1 /(2 \gamma+1)}$. For the other term in (15), we have

$$
\begin{aligned}
{\left[\log \left(1 / \sigma_{n}\right)\right]^{\frac{\gamma-\delta_{n}}{2\left(\gamma-\delta_{n}\right)+1}-\frac{\gamma}{2 \gamma+1}} } & =\left[\log \sigma^{-1}+(1 / 2) \log n\right]^{\mathcal{O}(1 / \log n)} \\
& =\exp \left(\mathcal{O}(1 / \log n) \log \left[\log \sigma^{-1}+(1 / 2) \log n\right]\right)
\end{aligned}
$$

which converges to one as $n \rightarrow \infty$. Thus, for this sequence $\delta_{n}$, the left-hand side of (15) converges to $\tilde{\varepsilon}^{-1 /(2 \gamma+1)}=\left(2 \bar{C} / \underline{C}_{K, \psi}\right)^{-1 /(2 \gamma+1)} \varepsilon^{-1 /(2 \gamma+1)}$. Since $\left(2 \bar{C} / \underline{C}_{K, \psi}\right)^{-1 /(2 \gamma+1)}$ is bounded from below by a positive constant uniformly over $\gamma \leq \bar{\gamma}$, it follows that (15) holds for this sequence $\delta_{n}$. This completes the proof of Theorem 2.2.

\section{Appendix: Details for Section 2.2}

This appendix provides details for the results in Section 2.2.

\section{A.1. Critical value}

The critical value $c(j)=\bar{c}_{K} \sigma_{n} 2^{j / 2} \sqrt{j}$ is justified by the following lemma.

Lemma A.1. Let $c(j)=\bar{c}_{K} \sigma_{n} 2^{j / 2} \sqrt{j}$ and suppose that (4) and (5) hold. Then, if $\bar{c}_{K}$ is larger than a constant that depends only on the kernel $K$, we will have, for any sequence $\underline{\ell}_{n} \rightarrow \infty$,

$$
P\left(\left|\hat{f}(t, j)-K_{j} f(t)\right| \leq c(j) \text { all } t \in[0,1], j \geq \underline{\ell}_{n}\right) \rightarrow 1 .
$$


Proof. Let $\mathbb{T}_{n}(t, j)=\sigma_{n}^{-1} 2^{-j / 2}\left[\hat{f}(t, j)-K_{j} f(t)\right]=\int 2^{j / 2} K\left(2^{j} t, 2^{j} x\right) d W(x)$. Note that the distribution of the process $t \mapsto \mathbb{T}_{n}\left(2^{-j}(t+k)\right)$ is the same for all $j, k, n$, since $\operatorname{cov}\left(\mathbb{T}_{n}\left(2^{-j}(s+\right.\right.$ $\left.k), j), \mathbb{T}_{n}\left(2^{-j}(t+k), j\right)\right)=\int 2^{j} K\left(s+k, 2^{j} x\right) K\left(t+k, 2^{j} x\right) d x=\int K(s, u) K(t, u) d u$, using change of variables $u=2^{j} x-k$ and the fact that $K(t+k, u+k)=K(t, u)$. Thus,

$$
\begin{aligned}
& P\left(\sup _{t \in[0,1]}\left|\mathbb{T}_{n}(t, j)\right|>\bar{c}_{K} \sqrt{j}\right) \\
& \quad \leq \sum_{k=0}^{2^{j}-1} P\left(\sup _{s \in[0,1]}\left|\mathbb{T}_{n}\left(2^{-j}(s+k), j\right)\right|>\bar{c}_{K} \sqrt{j}\right) \\
& \quad=2^{j} P\left(\sup _{t \in[0,1]}\left|\mathbb{T}_{n}(t, 1)\right|>\bar{c}_{K} \sqrt{j}\right) .
\end{aligned}
$$

By (5), we can apply Theorem 8.1 in [27] to the process $\mathbb{T}_{n}(t, 1)$, which, along with the tail bound $\Phi(-x) \leq(x \sqrt{2 \pi})^{-1} \exp \left(-x^{2} / 2\right)$ where $\Phi$ is the standard normal cdf, gives the bound $P\left(\sup _{t \in[0,1]}\left|\mathbb{T}_{n}(t, 1)\right|>\bar{c}_{K} \sqrt{j}\right) \leq C j^{1 / \tau_{K}-1} \exp \left(-j \bar{c}_{K} / C\right)$ for some constant $C$ that depends only on the kernel $K$. Thus,

$$
\begin{aligned}
1- & P\left(\left|\hat{f}(t, j)-K_{j} f(t)\right| \leq c(j) \text { all } t \in[0,1], j \geq \underline{\ell}_{n}\right) \\
& \leq \sum_{j=\underline{\ell}_{n}}^{\infty} 2^{j} P\left(\sup _{t \in[0,1]}\left|\mathbb{T}_{n}(t, 1)\right|>\bar{c}_{K} \sqrt{j}\right) \\
& \leq \sum_{j=\underline{\ell}_{n}}^{\infty} 2^{j} C j^{1 / \tau_{K}-1} \exp \left(-j \bar{c}_{K} / C\right)=\sum_{j=\underline{\ell}_{n}}^{\infty} C j^{1 / \tau_{K}-1} \exp \left(-j\left(\bar{c}_{K} / C-\log 2\right) .\right.
\end{aligned}
$$

For $\bar{c}_{K}>C \log 2$, this converges to 0 as $n \rightarrow \infty$.

\section{A.2. Confidence interval for $\gamma$}

We construct a confidence interval $\left[\hat{\gamma}_{\ell}, \hat{\gamma}_{u}\right]$ for $\gamma$, which can be used in the confidence band described in Section 2.2. The confidence interval covers $\gamma$ on the event in (7), so that the resulting confidence band for $f$ contains $f$ on the event that (6) and (7) both hold.

Let $\underline{G}\left(j_{1}, j_{2}\right)=\underline{G}\left(\varepsilon, \underline{B}, \bar{B}, \underline{\gamma}, \bar{\gamma}, j_{1}, j_{2}\right)=\min _{B \in[\underline{B}, \bar{B}], \gamma \in[\underline{\gamma}, \bar{\gamma}]} B\left(\varepsilon-2^{-\left(j_{2}-j_{1}\right) \gamma}\right)$ and $\bar{G}\left(j_{1}, j_{2}\right)=$ $\bar{G}\left(\underline{B}, \bar{B}, \underline{\gamma}, \bar{\gamma}, j_{1}, j_{2}\right)=\max _{B \in[\underline{B}, \bar{B}], \gamma \in[\underline{\gamma}, \bar{\gamma}]} B\left(1+2^{-\left(j_{2}-j_{1}\right) \gamma}\right)$. Let

$$
\tilde{\gamma}_{\ell}\left(j_{1}, j_{2}\right)=\frac{\log _{2} \underline{G}\left(j_{1}, j_{2}\right)-\log _{2}\left[\hat{\Delta}\left(j_{2}, j_{2}\right)+\tilde{c}\left(j_{1}, j_{2}\right)\right]}{j_{1}}
$$

with the convention that $\tilde{\gamma}_{\ell}\left(j_{1}, j_{2}\right)=\underline{\gamma}$ when $\underline{G}\left(j_{1}, j_{2}\right) \leq 0$. Let

$$
\tilde{\gamma}_{u}\left(j_{1}, j_{2}\right)=\frac{\log _{2} \bar{G}\left(j_{1}, j_{2}\right)-\log _{2}\left[\hat{\Delta}\left(j_{2}, j_{2}\right)-\tilde{c}\left(j_{1}, j_{2}\right)\right]}{j_{1}}
$$

with the convention that $\tilde{\gamma}_{u}\left(j_{1}, j_{2}\right)=\bar{\gamma}$ when $\log _{2}\left[\hat{\Delta}\left(j_{2}, j_{2}\right)-\tilde{c}\left(j_{1}, j_{2}\right)\right] \leq 0$. Let

$$
\hat{\gamma}_{\ell}=\max _{j \in \mathcal{J}_{n}} \tilde{\gamma}_{\ell}\left(j_{1}, j_{2}\right) \quad \text { and } \quad \hat{\gamma}_{u}=\min _{j \in \mathcal{J}_{n}} \tilde{\gamma}_{u}\left(j_{1}, j_{2}\right)
$$


Then $\gamma \in\left[\hat{\gamma}_{\ell}, \hat{\gamma}_{u}\right]$ on the event in (7). To see this, note that, by (8), we have, for all $j_{1}, j_{2} \in \mathcal{J}_{n}$

$$
2^{-j_{1} \gamma} \underline{G}\left(j_{1}, j_{2}\right) \leq 2^{-j_{1} \gamma} B\left(\varepsilon-2^{-\left(j_{2}-j_{1}\right) \gamma}\right) \leq \Delta\left(j_{1}, j_{2} ; f\right) \leq \hat{\Delta}\left(j_{1}, j_{2}\right)+\tilde{c}\left(j_{1}, j_{2}\right),
$$

and

$$
\hat{\Delta}\left(j_{2}, j_{2}\right)-\tilde{c}\left(j_{1}, j_{2}\right) \leq \Delta\left(j_{1}, j_{2} ; f\right) \leq 2^{-j_{1} \gamma} B\left(1+2^{-\left(j_{2}-j_{1}\right) \gamma}\right) \leq 2^{-j_{1} \gamma} \bar{G}\left(j_{1}, j_{2}\right) .
$$

Taking logs and rearranging gives $\gamma \in\left[\tilde{\gamma}_{\ell}\left(j_{1}, j_{2}\right), \tilde{\gamma}_{u}\left(j_{1}, j_{2}\right)\right]$. Note also that

$$
\begin{aligned}
& \tilde{\gamma}_{u}\left(j_{1}, j_{2}\right)-\tilde{\gamma}_{\ell}\left(j_{1}, j_{2}\right) \\
& \quad \leq \frac{\log _{2} \bar{G}\left(j_{1}, j_{2}\right)-\log _{2} \underline{G}\left(j_{1}, j_{2}\right)}{j_{1}}+\frac{2 \tilde{c}\left(j_{1}, j_{2}\right)}{j_{1}\left(\hat{\Delta}\left(j_{1}, j_{2}\right)-\tilde{c}\left(j_{1}, j_{2}\right)\right) \log 2} \\
& \quad \leq \frac{\log _{2} \bar{G}\left(j_{1}, j_{2}\right)-\log _{2} \underline{G}\left(j_{1}, j_{2}\right)}{j_{1}}+\frac{2 \tilde{c}\left(j_{1}, j_{2}\right)}{j_{1}\left(2^{-j_{1} \bar{\gamma}} \underline{G}\left(j_{1}, j_{2}\right)-2 \tilde{c}\left(j_{1}, j_{2}\right)\right) \log 2},
\end{aligned}
$$

where the first inequality uses $|\log a-\log b| \leq|a-b| / \min \{a, b\}$ and the second inequality uses (16).

Let $\tilde{c}\left(j_{1}, j_{2}\right)=\bar{c}_{K} \sigma_{n} 2^{j_{1} / 2} \sqrt{j_{1}}+\bar{c}_{K} \sigma_{n} 2^{j_{2} / 2} \sqrt{j_{2}}$, so that Lemma A.1 applies. Let $j_{1}, j_{2}$ satisfy $j_{1}, j_{2} \rightarrow \infty, j_{2}-j_{1} \rightarrow \infty$, and $j_{2} / \log n \rightarrow 0$. Then the above display is bounded by a constant times $j_{1}^{-1}$. To see this, note that $\underline{G}\left(j_{1}, j_{2}\right)$ and $\bar{G}\left(j_{1}, j_{2}\right)$ converge to positive constants, and $2^{j_{1}} \bar{\gamma} \tilde{c}\left(j_{1}, j_{2}\right) \rightarrow 0$ by the conditions on $j_{1}$ and $j_{2}$.

We collect these results in a theorem.

Theorem A.1. Let $\hat{\gamma}_{\ell}$ and $\hat{\gamma}_{u}$ be given above. Then, on the event in (7), we have $\gamma \in\left[\hat{\gamma}_{\ell}, \hat{\gamma}_{u}\right]$ for $f \in$ $\mathcal{F}_{\text {self-sim }}(\gamma, B, \varepsilon)$ with $B \in[\underline{B}, \bar{B}]$ and $\gamma \in[\underline{\gamma}, \bar{\gamma}]$. Furthermore, if we take $\tilde{c}\left(j_{1}, j_{2}\right)=\bar{c}_{K} \sigma_{n} 2^{j_{1} / 2} \sqrt{j_{1}}+$ $\bar{c}_{K} \sigma_{n} 2^{j_{2} / 2} \sqrt{j_{2}}$ and $\mathcal{J}_{n}$ contains sequences $\overline{j_{1}}=j_{1, n}$ and $j_{2}=j_{2, n}$ which satisfy $j_{1}, j_{2} \rightarrow \infty, j_{2}-j_{1} \rightarrow$ $\infty$, and $j_{2} / \log n \rightarrow 0$, then, for any sequence $r_{n}$ with $r_{n} \rightarrow 0$ and $r_{n} / j_{1} \rightarrow \infty$, we have

$$
\gamma-r_{n} \leq \hat{\gamma}_{\ell} \leq \gamma \leq \hat{\gamma}_{u} \leq \gamma+r_{n}
$$

with probability approaching one uniformly over $\bigcup_{\gamma \in[\underline{\gamma}, \bar{\gamma}], B \in[\underline{B}, \bar{B}]} \mathcal{F}_{\mathrm{GN}}(\varepsilon, \varepsilon B, B)$.

\section{A.3. Length of the confidence band}

We now bound the length of this confidence band. From (10), it follows that, on the event $\gamma-r_{n} \leq$ $\hat{\gamma}_{\ell} \leq \gamma \leq \hat{\gamma}_{u} \leq \gamma+r_{n}$, the length of the confidence band is bounded by

$$
\sup _{\gamma_{u}, \gamma_{\ell} \in\left[\gamma-r_{n}, \gamma+r_{n}\right]} \min _{j, j_{1}, j_{2} \in \mathcal{J}_{n}}\left[c(j)+\frac{B\left(2^{-j_{1} \gamma}+2^{-j_{2} \gamma}\right)+2 c\left(j_{1}\right)+2 c\left(j_{2}\right)}{a\left(\varepsilon, j_{1}, j_{2}, j, \gamma_{\ell}, \gamma_{u}\right)}\right],
$$

where $c(j)=\bar{c}_{K} \sigma 2^{j / 2} \sqrt{j / n}$.

It turns out that it will suffice to get an upper bound for the minimum in the above display by taking $j=j_{n, \gamma}=\left\lfloor\rho_{\gamma}+(2 \gamma+1)^{-1}\left(\log _{2}\left(n / \log _{2} n\right)\right)\right\rfloor, j_{1}=j_{1, n, \gamma}=j_{n, \gamma}-m_{1, n}$ and $j_{2}=j_{2, n, \gamma}=$ $j_{n, \gamma}-m_{2, n}$ where $m_{1, n}$ and $m_{2, n}$ are sequences such that $m_{2, n} \rightarrow \infty, m_{1, n}-m_{2, n} \rightarrow \infty, r_{n} m_{1, n} \rightarrow 0$ and, for all $\gamma \in[\underline{\gamma}, \bar{\gamma}], j_{1, n, \gamma} \rightarrow \infty$ and $j_{2, n, \gamma} \rightarrow \infty$. Applying the lemmas below gives the bound

$$
\left[\frac{\bar{c}_{K} \sigma 2^{\rho_{\gamma} / 2}}{(2 \gamma+1)^{1 / 2}}+B \varepsilon^{-1} 2^{\gamma\left(1-\rho_{\gamma}\right)}\right](n / \log n)^{-\gamma /(2 \gamma+1)}[1+o(1)]
$$


where the $o(1)$ term is over $\gamma \in[\underline{\gamma}, \bar{\gamma}], B \in[\underline{B}, \bar{B}]$. Setting $\rho_{\gamma}=\log _{2}\left(\sigma^{-1} B \varepsilon^{-1}\right)^{2 /(2 \gamma+1)}$ so that $2^{\rho_{\gamma} / 2}=\left(\sigma^{-1} B \varepsilon^{-1}\right)^{1 /(2 \gamma+1)}=\sigma^{2 \gamma /(2 \gamma+1)-1}\left(B \varepsilon^{-1}\right)^{1 /(2 \gamma+1)}$ gives

$$
\left[\frac{\bar{c}_{K}}{(2 \gamma+1)^{1 / 2}}+2^{\gamma}\right] \sigma^{2 \gamma /(2 \gamma+1)}\left(B \varepsilon^{-1}\right)^{1 /(2 \gamma+1)}(n / \log n)^{-\gamma /(2 \gamma+1)}[1+o(1)] .
$$

Since $\sigma_{n}^{2} \log \left(1 / \sigma_{n}\right)=\left(\sigma^{2} / n\right)((1 / 2) \log n-\log \sigma)=(1+o(1))\left(\sigma^{2} / 2\right)(\log n) / n$, this gives a bound of $\left(\sigma_{n}^{2} \log \left(1 / \sigma_{n}\right)\right)^{\gamma /(2 \gamma+1)}$ times a constant that is bounded uniformly over $\gamma \leq \bar{\gamma}$, as required.

\section{Lemma A.2.}

$$
\sup _{\gamma \in[\underline{\gamma}, \bar{\gamma}] \gamma_{\ell}, \gamma_{u} \in\left[\gamma-r_{n}, \gamma+r_{n}\right]}\left|\frac{a\left(\varepsilon, j_{1, n, \gamma}, j_{2, n, \gamma}, j_{n, \gamma}, \gamma_{\ell}, \gamma_{u}\right)}{a\left(\varepsilon, j_{1, n, \gamma}, j_{2, n, \gamma}, j_{n, \gamma}, \gamma, \gamma\right)}-1\right| \rightarrow 0
$$

Proof. For $n$ large enough, we have, for any $\gamma \in[\underline{\gamma}, \bar{\gamma}]$ and $\gamma_{\ell}, \gamma_{u}$ with $\gamma-r_{n} \leq \gamma_{\ell} \leq \gamma_{u} \leq \gamma+r_{n}$,

$$
\varepsilon 2^{m_{1, n}\left(\gamma-r_{n}\right)}-2^{m_{2, n}\left(\gamma+r_{n}\right)} \leq a\left(\varepsilon, j_{1, n, \gamma}, j_{2, n, \gamma}, j_{n, \gamma}, \gamma_{\ell}, \gamma_{u}\right) \leq \varepsilon 2^{m_{1, n}\left(\gamma+r_{n}\right)}-2^{m_{2, n}\left(\gamma-r_{n}\right)}
$$

and $a\left(\varepsilon, j_{1, n, \gamma}, j_{2, n, \gamma}, j_{n, \gamma}, \gamma, \gamma\right)=\varepsilon 2^{m_{1, n} \gamma}-2^{m_{2, n} \gamma}$. Thus,

$$
\begin{aligned}
\frac{a\left(\varepsilon, j_{1, n, \gamma}, j_{2, n, \gamma}, j_{n, \gamma}, \gamma_{\ell}, \gamma_{u}\right)}{a\left(\varepsilon, j_{1, n, \gamma}, j_{2, n, \gamma}, j_{n, \gamma}, \gamma, \gamma\right)} & \leq \frac{\varepsilon 2^{m_{1, n}\left(\gamma+r_{n}\right)}-2^{m_{2, n}\left(\gamma-r_{n}\right)}}{\varepsilon 2^{m_{1, n} \gamma}-2^{m_{2, n} \gamma}} \\
& =\frac{2^{m_{1, n} r_{n}}-\varepsilon^{-1} 2^{-m_{2, n} r_{n}+\left(m_{2, n}-m_{1, n}\right) \gamma}}{1-\varepsilon^{-1} 2^{\left(m_{2, n}-m_{1, n}\right) \gamma}},
\end{aligned}
$$

which converges to one uniformly over $\gamma \in[\underline{\gamma}, \bar{\gamma}]$ by the conditions on $m_{1, n}$ and $m_{2, n}$. The result follows from this and a similar argument with the lower bound.

\section{Lemma A.3.}

$$
\frac{2^{-\gamma j_{1, n, \gamma}}+2^{-\gamma j_{2, n, \gamma}}}{a\left(\varepsilon, j_{1, n, \gamma}, j_{2, n, \gamma}, j_{n, \gamma}, \gamma, \gamma\right)}=2^{-\gamma j_{n, \gamma}} \varepsilon^{-1}(1+o(1)),
$$

where the o(1) term is uniform over all $\gamma \in[\underline{\gamma}, \bar{\gamma}]$.

Proof. We have

$$
\begin{gathered}
\frac{2^{-\gamma j_{1, n, \gamma}}+2^{-\gamma j_{2, n, \gamma}}}{2^{-\gamma j_{n, \gamma}} \varepsilon^{-1} a\left(\varepsilon, j_{1, n, \gamma}, j_{2, n, \gamma}, j_{n, \gamma}, \gamma, \gamma\right)} \\
=\frac{2^{-\gamma\left(j_{1, n, \gamma}-j_{n, \gamma}\right)}+2^{-\gamma\left(j_{2, n, \gamma}-j_{n, \gamma}\right)}}{2^{m_{1, n} \gamma}-\varepsilon^{-1} 2^{m_{2, n} \gamma}} \\
=\frac{1+2^{-\left(m_{1, n}-m_{2, n}\right) \gamma}}{1-\varepsilon^{-1} 2^{-\left(m_{1, n}-m_{2, n}\right) \gamma}},
\end{gathered}
$$

which converges to one uniformly over $\gamma \in[\underline{\gamma}, \bar{\gamma}]$ by the conditions on $m_{1, n}$ and $m_{2, n}$. 
Lemma A.4. If $\rho_{\gamma}$ is bounded over $\gamma \in[\underline{\gamma}, \bar{\gamma}]$, then $c\left(j_{1, n, \gamma}\right) / 2^{-\gamma j_{1, n, \gamma}} \rightarrow 0$ and $c\left(j_{2, n, \gamma}\right) / 2^{-\gamma j_{2, n, \gamma} \rightarrow}$ 0 uniformly over $\gamma \in[\gamma, \bar{\gamma}]$. Furthermore, $c\left(j_{n, \gamma}\right) \leq \bar{c}_{K} \sigma 2^{\rho_{\gamma} / 2}(2 \gamma+1)^{-1 / 2}(n / \log n)^{-\gamma /(2 \gamma+1)}$ and $2^{-\gamma j_{n, \gamma}} \leq 2^{\gamma\left(1-\rho_{\gamma}\right)}\left(n / \log _{2} n\right)^{-\gamma /(2 \gamma+1)}$.

Proof. We have

$$
\begin{aligned}
c\left(j_{n, \gamma}\right)^{2} /\left(\bar{c}_{K} \sigma\right)^{2} & =2^{j_{n, \gamma}} j_{n, \gamma} / n \\
& =2^{\left\lfloor\rho_{\gamma}+(2 \gamma+1)^{-1}\left(\log _{2}\left(n / \log _{2} n\right)\right)\right\rfloor}\left\lfloor(2 \gamma+1)^{-1}\left(\log _{2} n-\log _{2} \log _{2} n\right)\right\rfloor / n \\
& \leq 2^{\rho_{\gamma}} 2^{(2 \gamma+1)^{-1}\left(\log _{2}\left(n / \log _{2} n\right)\right)}(2 \gamma+1)^{-1}\left(\log _{2} n\right) / n \\
& =2^{\rho_{\gamma}}(2 \gamma+1)^{-1}\left(n / \log _{2} n\right)^{-2 \gamma /(2 \gamma+1)}
\end{aligned}
$$

and

$$
\begin{gathered}
2^{-\gamma j_{n, \gamma}}=2^{-\gamma\left\lfloor\rho_{\gamma}+(2 \gamma+1)^{-1} \log _{2}\left(n / \log _{2} n\right)\right\rfloor} \\
\quad \leq 2^{\gamma\left(1-\rho_{\gamma}\right)-\gamma(2 \gamma+1)^{-1} \log _{2}\left(n / \log _{2} n\right)} \\
=2^{\gamma\left(1-\rho_{\gamma}\right)}\left(n / \log _{2} n\right)^{\gamma /(2 \gamma+1)}
\end{gathered}
$$

For any $m \geq \rho_{\gamma}$, we have

$$
\begin{aligned}
c\left(j_{n, \gamma}-m\right)^{2} /\left(2^{-\gamma\left(j_{n, \gamma}-m\right)} \bar{c}_{K} \sigma\right)^{2} & =2^{(2 \gamma+1)\left(j_{n, \gamma}-m\right)}\left(j_{n, \gamma}-m\right) / n \\
& \leq 2^{\log _{2}\left(n / \log _{2} n\right)-\left(m-\rho_{\gamma}\right)(2 \gamma+1)}(2 \gamma+1)^{-1}\left(\log _{2} n\right) / n \\
& =2^{-\left(m-\rho_{\gamma}\right)(2 \gamma+1)}(2 \gamma+1)^{-1} .
\end{aligned}
$$

Setting $m=m_{1, n} \rightarrow \infty$ it follows that $c\left(j_{1, n, \gamma}\right) / 2^{-\gamma j_{1, n, \gamma}} \rightarrow 0$ uniformly over $\gamma \in[\underline{\gamma}, \bar{\gamma}]$ and similarly for $j_{2, n, \gamma}$.

\section{Acknowledgements}

Thanks to Richard Nickl for helpful comments and discussion, and to the anonymous referees for helpful suggestions.

\section{References}

[1] Armstrong, T.B. and Kolesár, M. (2020). Simple and honest confidence intervals in nonparametric regression. Quant. Econ. 11 1-39. MR4084655 https://doi.org/10.3982/qe1199

[2] Bahadur, R.R. and Savage, L.J. (1956). The nonexistence of certain statistical procedures in nonparametric problems. Ann. Math. Stat. 27 1115-1122. MR0084241 https://doi.org/10.1214/aoms/1177728077

[3] Brown, L.D. and Low, M.G. (1996). Asymptotic equivalence of nonparametric regression and white noise. Ann. Statist. 24 2384-2398. MR1425958 https://doi.org/10.1214/aos/1032181159

[4] Bühlmann, P. and van de Geer, S. (2011). Statistics for High-Dimensional Data: Methods, Theory and Applications. Springer Series in Statistics. Heidelberg: Springer. MR2807761 https://doi.org/10.1007/ 978-3-642-20192-9 
[5] Bull, A.D. (2012). Honest adaptive confidence bands and self-similar functions. Electron. J. Stat. 6 14901516. MR2988456 https://doi.org/10.1214/12-EJS720

[6] Bull, A.D. and Nickl, R. (2013). Adaptive confidence sets in $L^{2}$. Probab. Theory Related Fields 156889 919. MR3078289 https://doi.org/10.1007/s00440-012-0446-z

[7] Cai, T.T. and Low, M.G. (2004). An adaptation theory for nonparametric confidence intervals. Ann. Statist. 32 1805-1840. MR2102494 https://doi.org/10.1214/009053604000000049

[8] Cai, T.T. and Low, M.G. (2006). Adaptive confidence balls. Ann. Statist. 34 202-228. MR2275240 https://doi.org/10.1214/009053606000000146

[9] Carpentier, A. (2013). Honest and adaptive confidence sets in $L_{p}$. Electron. J. Stat. 7 2875-2923. MR3148371 https://doi.org/10.1214/13-EJS867

[10] Chernozhukov, V., Chetverikov, D. and Kato, K. (2014). Anti-concentration and honest, adaptive confidence bands. Ann. Statist. 42 1787-1818. MR3262468 https://doi.org/10.1214/14-AOS1235

[11] Donoho, D.L. (1994). Statistical estimation and optimal recovery. Ann. Statist. 22 238-270. MR1272082 https://doi.org/10.1214/aos/1176325367

[12] Giné, E. and Nickl, R. (2010). Confidence bands in density estimation. Ann. Statist. 38 1122-1170. MR2604707 https://doi.org/10.1214/09-AOS738

[13] Giné, E. and Nickl, R. (2016). Mathematical Foundations of Infinite-Dimensional Statistical Models, 1st ed. Cambridge Series in Statistical and Probabilistic Mathematics. New York: Cambridge Univ. Press. MR3588285 https://doi.org/10.1017/CBO9781107337862

[14] Hoffmann, M. and Nickl, R. (2011). On adaptive inference and confidence bands. Ann. Statist. 39 23832409. MR2906872 https://doi.org/10.1214/11-AOS903

[15] Ingster, Y.I. and Suslina, I.A. (2003). Nonparametric Goodness-of-Fit Testing Under Gaussian Models. Lecture Notes in Statistics 169. New York: Springer. MR1991446 https://doi.org/10.1007/978-0-387-21580-8

[16] Juditsky, A. and Lambert-Lacroix, S. (2003). Nonparametric confidence set estimation. Math. Methods Statist. 12 410-428. MR2054156

[17] Knafl, G., Sacks, J. and Ylvisaker, D. (1982). Model robust confidence intervals. J. Statist. Plann. Inference 6 319-334. MR0667912 https://doi.org/10.1016/0378-3758(82)90002-7

[18] Kueh, A. (2012). Locally adaptive density estimation on the unit sphere using needlets. Constr. Approx. 36 433-458. MR2996439 https://doi.org/10.1007/s00365-012-9170-2

[19] Lepski, O.V. and Tsybakov, A.B. (2000). Asymptotically exact nonparametric hypothesis testing in supnorm and at a fixed point. Probab. Theory Related Fields 117 17-48. MR1759508 https://doi.org/10.1007/ s004400050265

[20] Low, M.G. (1997). On nonparametric confidence intervals. Ann. Statist. 25 2547-2554. MR1604412 https://doi.org/10.1214/aos/1030741084

[21] Mukherjee, R. and Sen, S. (2018). Optimal adaptive inference in random design binary regression. Bernoulli 24 699-739. MR3706774 https://doi.org/10.3150/16-BEJ893

[22] Nickl, R. and Szabó, B. (2016). A sharp adaptive confidence ball for self-similar functions. Stochastic Process. Appl. 126 3913-3934. MR3565485 https://doi.org/10.1016/j.spa.2016.04.017

[23] Nickl, R. and van de Geer, S. (2013). Confidence sets in sparse regression. Ann. Statist. 41 2852-2876. MR3161450 https://doi.org/10.1214/13-AOS1170

[24] Nussbaum, M. (1996). Asymptotic equivalence of density estimation and Gaussian white noise. Ann. Statist. 24 2399-2430. MR1425959 https://doi.org/10.1214/aos/1032181160

[25] Patschkowski, T. and Rohde, A. (2019). Locally adaptive confidence bands. Ann. Statist. 47 349-381. MR3909936 https://doi.org/10.1214/18-AOS1690

[26] Picard, D. and Tribouley, K. (2000). Adaptive confidence interval for pointwise curve estimation. Ann. Statist. 28 298-335. MR1762913 https://doi.org/10.1214/aos/1016120374

[27] Piterbarg, V.I. (1996). Asymptotic Methods in the Theory of Gaussian Processes and Fields. Translations of Mathematical Monographs 148. Providence, RI: Amer. Math. Soc. MR1361884 https://doi.org/10.1090/ mmono/148

[28] Robins, J. and van der Vaart, A. (2006). Adaptive nonparametric confidence sets. Ann. Statist. 34 229-253. MR2275241 https://doi.org/10.1214/009053605000000877

[29] Sniekers, S. and van der Vaart, A. (2015). Adaptive Bayesian credible sets in regression with a Gaussian process prior. Electron. J. Stat. 9 2475-2527. MR3425364 https://doi.org/10.1214/15-EJS1078 
[30] Szabó, B., van der Vaart, A.W. and van Zanten, J.H. (2015). Frequentist coverage of adaptive nonparametric Bayesian credible sets. Ann. Statist. 43 1391-1428. MR3357861 https://doi.org/10.1214/14-AOS1270

[31] Tsybakov, A.B. (1998). Pointwise and sup-norm sharp adaptive estimation of functions on the Sobolev classes. Ann. Statist. 26 2420-2469. MR1700239 https://doi.org/10.1214/aos/1024691478

[32] van der Pas, S., Szabó, B. and van der Vaart, A. (2017). Uncertainty quantification for the horseshoe (with discussion). Bayesian Anal. 12 1221-1274. MR3724985 https://doi.org/10.1214/17-BA1065

Received February 2020 and revised September 2020 